Supporting Information for

\title{
Dynamic Transformation between Covalent Organic Frameworks and Discrete Organic Cages
}

Zhen Shan, ${ }^{\dagger,}$ Xiaowei Wu, ${ }^{\dagger}{ }^{\S}$ Bingqing Xu, ${ }^{\dagger}$ You-lee Hong, ${ }^{\ddagger}, \mathbb{l}$ Miaomiao Wu, ${ }^{\dagger}$ Yuxiang Wang, ${ }^{\dagger}$ Yusuke Nishiyama, ${ }^{\ddagger}, \perp$ Junwu Zhu ${ }^{\dagger}$ Satoshi Horike, ${ }^{\mathbb{I}}$ Susumu Kitagawa ${ }^{\mathbb{I}}$ and Gen Zhang ${ }^{*, \dagger}$

${ }^{\dagger}$ Key Laboratory for Soft Chemistry and Functional Materials of Ministry of Education, School of Chemical Engineering, Nanjing University of Science and Technology, Nanjing, Jiangsu 210094, China.

$\$$ RIKEN-JEOL Collaboration Center, and RIKEN SPring-8 Center, Tsurumi, Yokohama, Kanagawa 230-0045, Japan

${ }^{\perp}$ JEOL RESONANCE Inc., 3-1-2 Musashino, Akishima, Tokyo 196-8558, Japan.

${ }^{\mathbb{I}}$ Institute for Integrated Cell-Material Sciences, Institute for Advanced Study, Kyoto University, Yoshida, Sakyo-ku Kyoto 606-8501, Japan.

$\S$ Z.S. and X.W. contributed equally.

E-mail: zhanggen@njust.edu.cn 


\section{Table of Content}

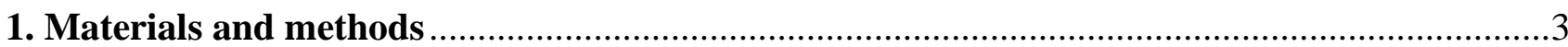

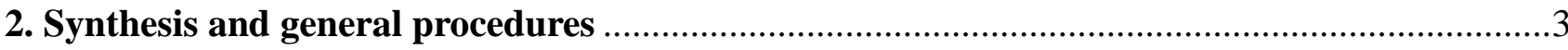

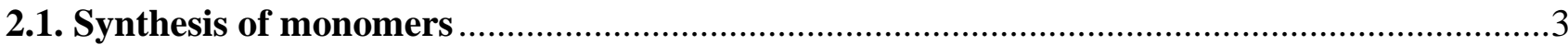

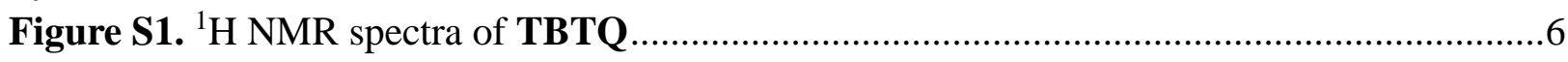

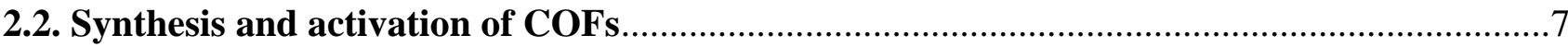

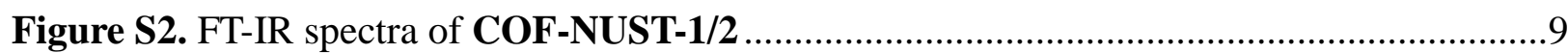

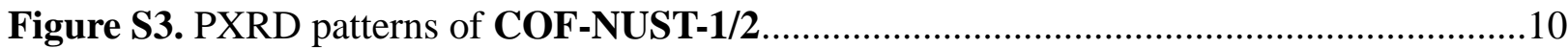

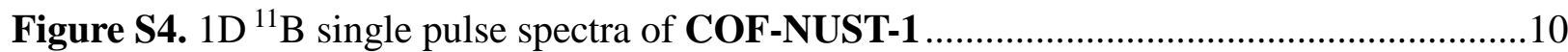

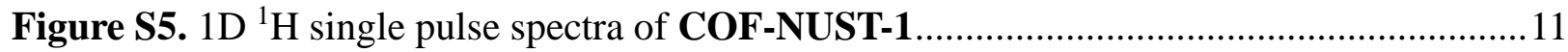

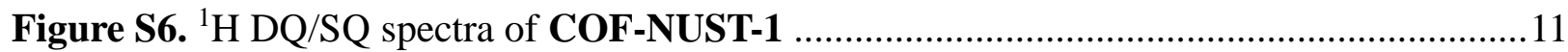

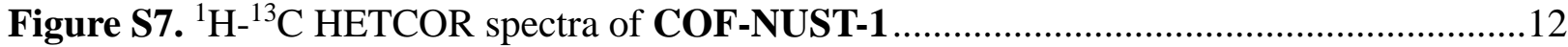

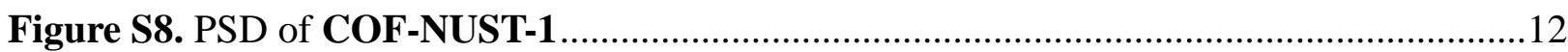

Figure S9. Simulated PXRD patterns of COF-NUST-1 …..................................................13

Figure S10. PXRD pattern of COF-LZU-1 …............................................................ 15

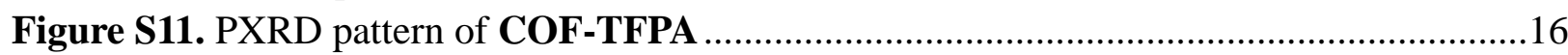

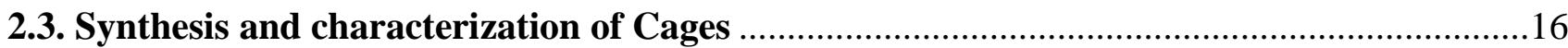

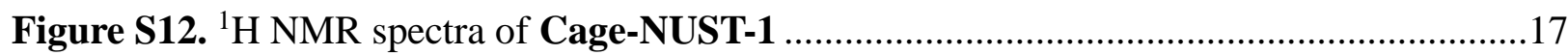

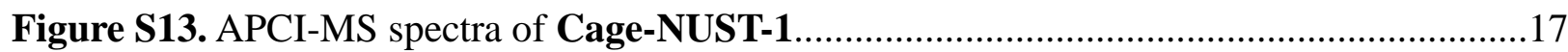

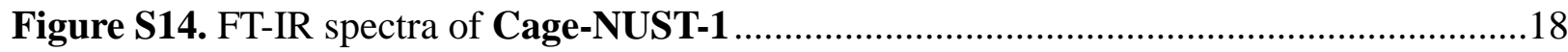

Figure S15. ${ }^{1} \mathrm{H}$ NMR spectra of Cage 1 ........................................................................... 19

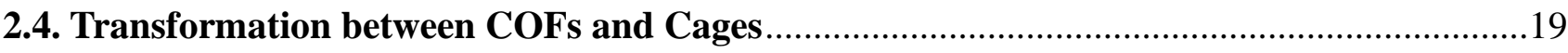

Figure S16. FT-IR spectra of COF-NUST-1, Cage-NUST-1 …............................................20

Figure S17. ${ }^{1} \mathrm{H}$ NMR spectra of Cage-NUST-1 from COF-NUST-1/2 ..................................22

Figure S18. ${ }^{1} \mathrm{H}$ NMR spectra of the compounds from Cage-NUST-1 to COF-NUST-1...........23

Figure S19. PXRD patterns of compound from Cage-NUST-1 to COF-NUST-1 ..................23

Figure S20. ${ }^{1}$ H NMR spectra of Cage-NUST-1 from Self-Sorting .......................................24

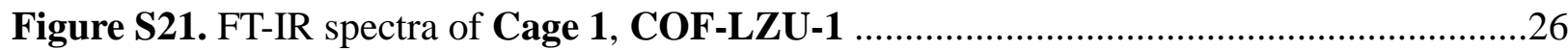

Figure S22. PXRD patterns of COF-LZU-1 from Cage 1 .................................................27

Figure S23. PXRD patterns of COF-TFPA from Cage 1 ....................................................28

Figure S24. $\mathrm{N}_{2}$ adsorption-desorption isotherms of COF-TFPA from Cage 1 ......................29

Figure S25. PXRD patterns of the transformation from COF-LZU-1 to Cage 1 .....................30

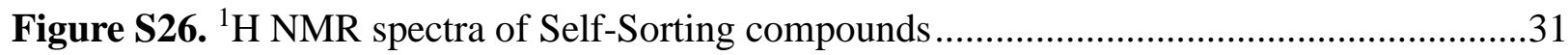

Figure S27. PXRD patterns of precipitate of Self-Sorting .....................................................32

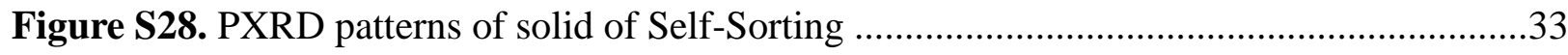

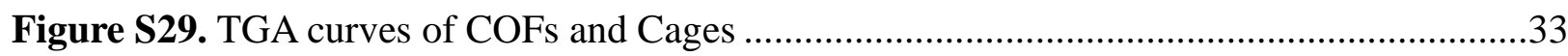

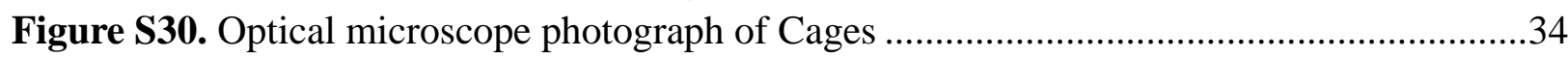

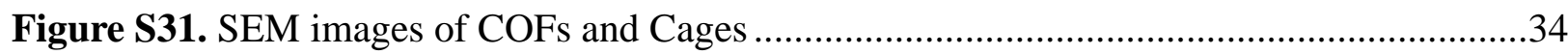

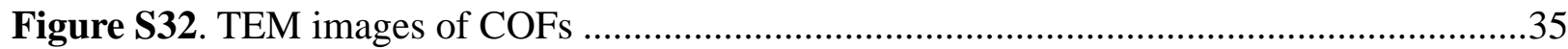

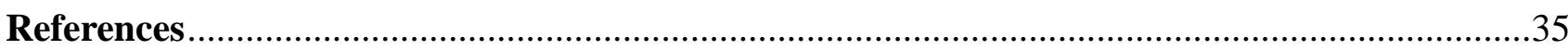




\section{Materials and methods}

All the chemicals are commercially available, and used without further purification. All solvents were dried and distilled according to conventional methods.

Powder X-ray diffraction (PXRD): PXRD patterns were collected on a Bruker D8 Advance diffractometer using $\mathrm{Cu} \mathrm{K} \alpha$ radiation.

Fourier transform infrared (FT-IR): IR spectrum was measured on a Thermo Fisher Scientific Optics NICOLETIS10 FT-IR spectrometer with Universal ATR accessory between the ranges of 4000 to $500 \mathrm{~cm}^{-1}$.

Solution nuclear magnetic resonance (NMR): Liquid state ${ }^{1} \mathrm{H}$ nuclear magnetic resonance spectroscopy were collected on a Bruker Avance III instrument with AS500 magnet equipped with a cryoprobe $(500 \mathrm{MHz})$.

Solid-state nuclear magnetic resonance (sSNMR): All ssNMR spectra were recorded on a JEOL JNM-ECZ600R spectrometer at $14.1 \mathrm{~T}\left({ }^{1} \mathrm{H}: 600 \mathrm{MHz}\right)$ and $1.0 \mathrm{~mm}$ rotors. $1 \mathrm{D}{ }^{1} \mathrm{H}$ and $2 \mathrm{D}{ }^{1} \mathrm{H}_{-}{ }^{13} \mathrm{C}$ heteronuclear correlation ssNMR spectra were measured at MAS of $70 \mathrm{kHz}$ and a recycle delay of $2.5 \mathrm{sec} .1 \mathrm{D}{ }^{11} \mathrm{~B}$ single-pulse spectrum was measured at MAS of $30 \mathrm{kHz}$ and recycle delay of $3 \mathrm{sec}$. $\pi / 2$ pulse lengths of ${ }^{1} \mathrm{H},{ }^{13} \mathrm{C}$ and ${ }^{11} \mathrm{~B}$ are $0.8 \mu \mathrm{s}, 1.15 \mu \mathrm{s}$, and $5.8 \mu \mathrm{s}$, respectively.

High resolution mass spectrometry (HRMS): HRMS mass spectra were collected on a Baird Acquity UPLC/XEVO G2-XS QTOF using $\mathrm{CHCl}_{3}$ as a solvent.

Scanning electron microscope (SEM): SEM images were collected using a JSM-IT500HR system.

Transmission electron microscope (TEM): TEM images were obtained with a JEM-2100, JEOL.

Gas adsorption: Gas adsorption measurement $\mathrm{N}_{2}$ adsorption and desorption measurements were performed at $77 \mathrm{~K}$ using ASAP 2020, Micromeritics Instrument Corp, USA. Pore size distributions and pore volumes were derived from the adsorption isotherms.

Thermogravimetric analysis (TGA): TGA was performed using a NETZSCH STA 449F5 under flowing $\mathrm{N}_{2}$ with $10 \mathrm{~K} \mathrm{~min}^{-1}$ ramp rate. Samples were heated in a Platinum pan $\left(900{ }^{\circ} \mathrm{C}, 10{ }^{\circ} \mathrm{C} \mathrm{min}^{-1}\right.$ ) under a $\mathrm{N}_{2}$ flux $\left(60 \mathrm{~mL} \mathrm{~min}^{-1}\right)$.

Optical microscopy images were collected on a UMT203i R/T Metallurgical Microscope made in Chongqing Aopu Optoelectronic Technology Co., Ltd, China.

\section{Synthesis and general procedures}

\subsection{Synthesis of monomers}

\subsubsection{Synthesis of Bis(3,4-dimethoxyphenyl)methanol (1)}

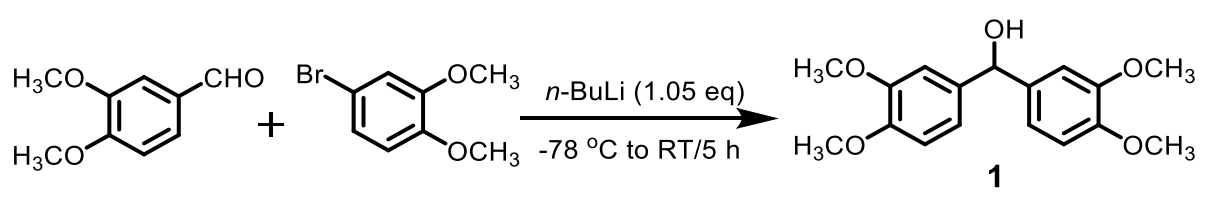

Compound $\mathbf{1}$ was synthesized according to the reported literature with a modified procedure. ${ }^{\mathbf{S 1}}$ 
$n$-BuLi (2.4 M in hexane, $4.4 \mathrm{~mL})$ was added dropwise to the solution of 4-bromoveratrole $(2.17 \mathrm{~g}$, $0.01 \mathrm{~mol})$ in dry THF $(40 \mathrm{~mL})$ at $-78{ }^{\circ} \mathrm{C}$. After $30 \mathrm{~min}$, a solution of 3,4-dimethoxybenzaldehyde $(1.66 \mathrm{~g}, 0.01 \mathrm{~mol})$ in dry THF $(10 \mathrm{~mL})$ was added dropwise at $-78^{\circ} \mathrm{C}$. Then the reaction mixture was warmed up slowly and the mixture was stirred at $22{ }^{\circ} \mathrm{C}$ for $5 \mathrm{~h}$. The reaction was quenched by cautiously adding saturated aqueous $\mathrm{NH}_{4} \mathrm{Cl}(20 \mathrm{~mL})$ and kept stirred at $22{ }^{\circ} \mathrm{C}$ for 30 min before extraction with diethyl ether $(3 \times 50 \mathrm{~mL})$. The reaction was quenched with methanol and extracted with $\mathrm{CH}_{2} \mathrm{Cl}_{2}(3 \times 30 \mathrm{~mL})$. The organic layer was washed with water $(3 \times 35 \mathrm{~mL})$ and then dried over $\mathrm{MgSO}_{4}$. After removing the solvent under reduced pressure and the residue was obtained as a pale-yellow foamy solid, compound 1 (2.34 g, 77\%), which was used for the next step without further purification.

\subsubsection{Synthesis of 5,6-Dimethoxy-2-methyl-1-indene-1,3-dione (2)}

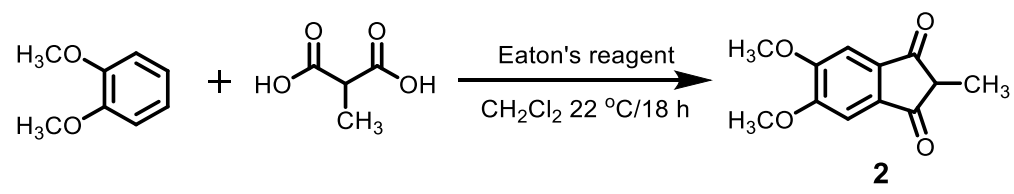

Compound 2 was synthesized according to the reported literature with a modified procedure. ${ }^{\mathbf{S 1}}$ $25 \mathrm{~mL}$ dichloromethane was added to a $100 \mathrm{~mL}$ round-bottom flask containing the mixture 1,2-dimethoxybenzene $(1.38 \mathrm{~g}, 0.01 \mathrm{~mol})$ and methylmalonic acid $(1.42 \mathrm{~g}, 0.01 \mathrm{~mol})$. Eaton's reagent $(15 \mathrm{~mL}, 9.1 \mathrm{w} \%$ in methanesulfonic acid) was added to the round-bottom flask and the mixture was stirred at $22{ }^{\circ} \mathrm{C}$ for $18 \mathrm{~h}$. The mixture was poured into ice water $(100 \mathrm{ml})$ and the product was extracted into DCM $(3 \times 50 \mathrm{ml})$. The solvent was removed under reduced pressure and the crude product was washed with $\mathrm{MeOH}$ to afford a pale yellow solid, compound $2(2.0 \mathrm{~g}, 91 \%)$, which was used for the next step without further purification.

\subsubsection{Synthesis of 2-[Bis(3,4-dimethoxyphenyl)methyl]-5,6-dimethoxy-2-methyl-1-in-dene}

\section{-1,3-dione (3)}

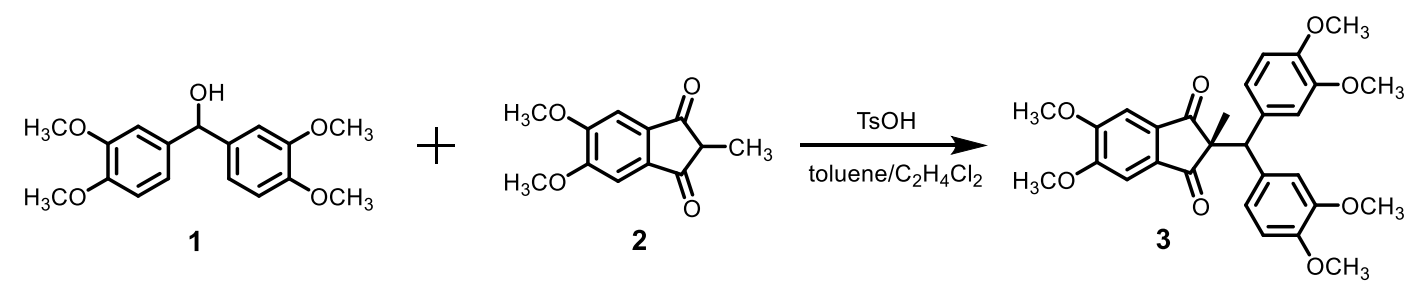

Compound $\mathbf{3}$ was synthesized according to the reported literature with a modified procedure. ${ }^{\mathbf{S 1}}$ A solution of compound 1 (2.26 g, $4.1 \mathrm{~mol})$ in dichloroethane $(30 \mathrm{~mL})$ was added dropwise into the mixture of compound $2(1.27 \mathrm{~g}, 5.76 \mathrm{~mol})$ and $p$-toluenesulfonic acid monohydrate $(0.11 \mathrm{~g}, 0.64 \mathrm{~mol})$ 
in toluene $(50 \mathrm{~mL})$ at refluxing state over $1 \mathrm{~h}$. The mixture was continued heating for $2 \mathrm{~h}$ and then allowed to cool. The solvent was removed under reduced pressure and the residue was re-dissolved in $\mathrm{CH}_{2} \mathrm{Cl}_{2}(100 \mathrm{~mL})$, and washed with saturated aqueous $\mathrm{Na}_{2} \mathrm{CO}_{3}(3 \times 50 \mathrm{~mL})$ and water $(100 \mathrm{~mL})$, dried over anhydrous $\mathrm{MgSO}_{4}$, removed solvent under reduced pressure and the crude product was washed with $\mathrm{MeOH}$ to afford a pale yellow solid as compound $\mathbf{3}(1.89 \mathrm{~g}, 65 \%)$, which was used for the next step without further purification.

2.1.4 Synthesis of Bis(3,4-dimethoxyphenyl)methyl-5,6-dimethoxy-2-methyl-1,3-inda- nediol (4)

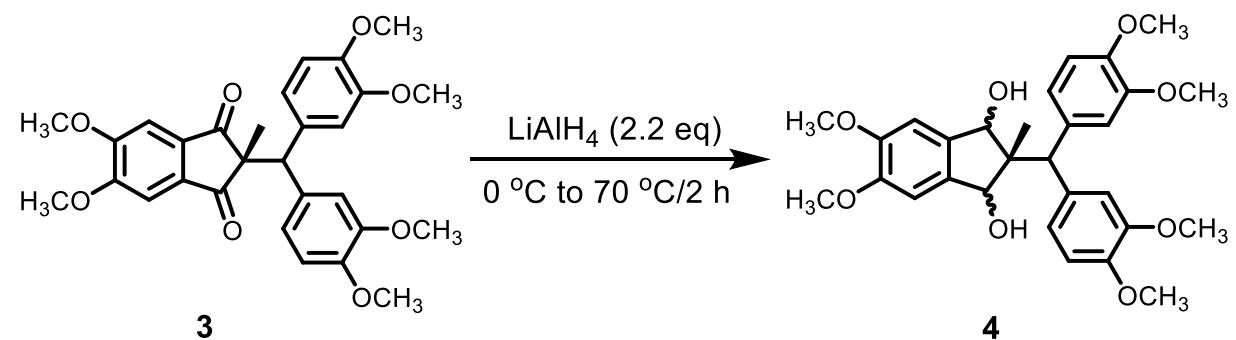

Compound $\mathbf{4}$ was synthesized according to the reported literature with a modified procedure. ${ }^{\mathbf{S 1}}$ Lithium aluminium hydride $(6.2 \mathrm{~mL}, 1 \mathrm{M}$ in THF) was added dropwise into a solution of compound $3(1.42 \mathrm{~g}, 2.8 \mathrm{mmol})$ in dry THF $(25 \mathrm{~mL})$ at $0{ }^{\circ} \mathrm{C}$. The reaction mixture was warmed up slowly and then heated to reflux for $2 \mathrm{~h}$. Then the mixture was cooled to $0{ }^{\circ} \mathrm{C}$, water $(0.5 \mathrm{~mL})$ and aqueous $\mathrm{NaOH}(1 \mathrm{M}, 0.5 \mathrm{~mL})$ were added dropwise subsequently after diluted with diethyl ether $(20 \mathrm{~mL})$. The mixture was warmed to room temperature and stirred for $1 \mathrm{~h}$ and then dried over anhydrous $\mathrm{Na}_{2} \mathrm{SO}_{4}$, filtered through a short pad of silica gel, rinsed with diethyl ether, concentrated under reduced pressure to afford compound 4, a pale yellow foamy solid (1.41 $\mathrm{g}, 98 \%)$.

\subsubsection{Synthesis of $2,3,6,7,10,11-H e x a m e t h o x y-12 d-$ methyltribenzotriquinacene (5)}

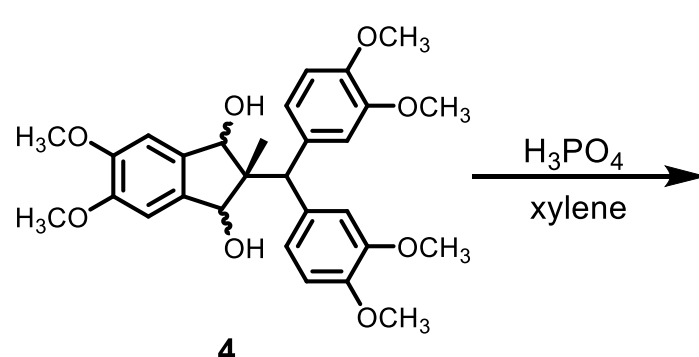

4

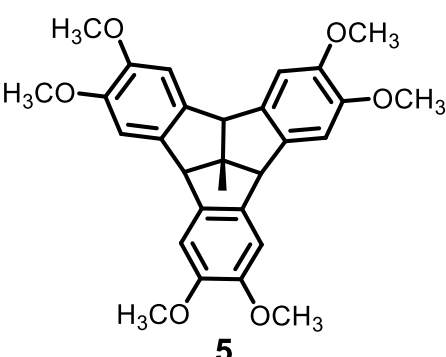

Compound $\mathbf{5}$ was synthesized according to the reported literature with a modified procedure. ${ }^{\mathbf{S 1}}$ A mixture of orthophosphoric acid $(85 \%, 25 \mathrm{~mL})$ and xylene $(20 \mathrm{~mL})$ was heated to reflux for 30 min in a $100 \mathrm{~mL}$ double round bottom flask. A solution of compound 4 (1.14 g, $2.23 \mathrm{mmol})$ in xylene $(10 \mathrm{~mL})$ was added dropwise. Through a constant pressure drop funnel over $30 \mathrm{~min}$. After the 
addition was complete, kept reflux for $4 \mathrm{~h}$. The mixture was cooled to room temperature and concentrated under reduced pressure to obtain a brown solid, which was recrystallized with $\mathrm{MeOH}$ to afford compound 5 (0.62 g, 55\%), a pale yellow solid.

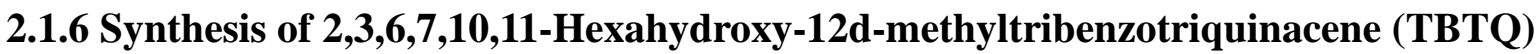

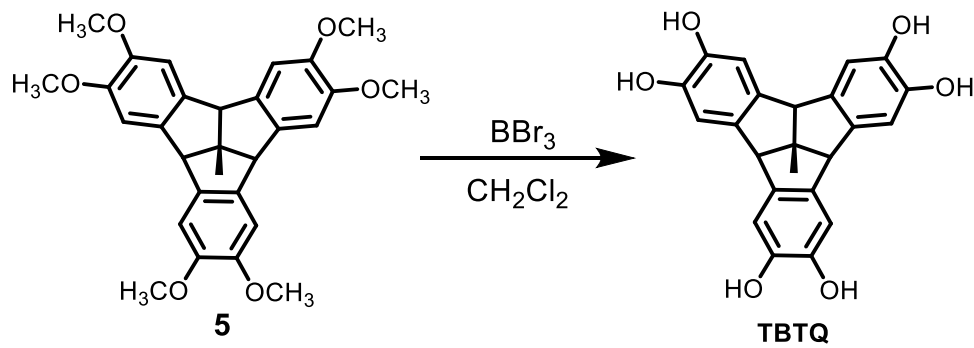

TBTQ was synthesized according to the reported literature with a modified procedure. ${ }^{\mathbf{S 1}}$ Boron tribromide (1 $\mathrm{M}$ in $\left.\mathrm{CH}_{2} \mathrm{Cl}_{2}, 18 \mathrm{~mL}\right)$ was added dropwise to a solution of 5 (1.2 $\left.\mathrm{g}, 2.5 \mathrm{mmol}\right)$ in anhydrous dichloromethane $(50 \mathrm{~mL})$ at $0{ }^{\circ} \mathrm{C}$. The reaction mixture was warmed up to room temperature for $5 \mathrm{~h}$, and then quenched by water $(25 \mathrm{~mL})$ at $0{ }^{\circ} \mathrm{C}$. The solvent was evaporated under reduced pressure, and extracted with ethyl acetate $(3 \times 50 \mathrm{~mL})$. The organic phase was dried over anhydrous $\mathrm{MgSO}_{4}$ and concentrated under reduced pressure to afford compound TBTQ (0.69 g, 70\%), a light-purple solid, which was used for the next step without further purification. ${ }^{1} \mathbf{H}$ NMR (300 MHz, CD $\left.{ }_{3} \mathrm{OD}\right): \delta 6.76$ (s, 6H), 4.01 (s, 3H), 1.53 (s, 3H).

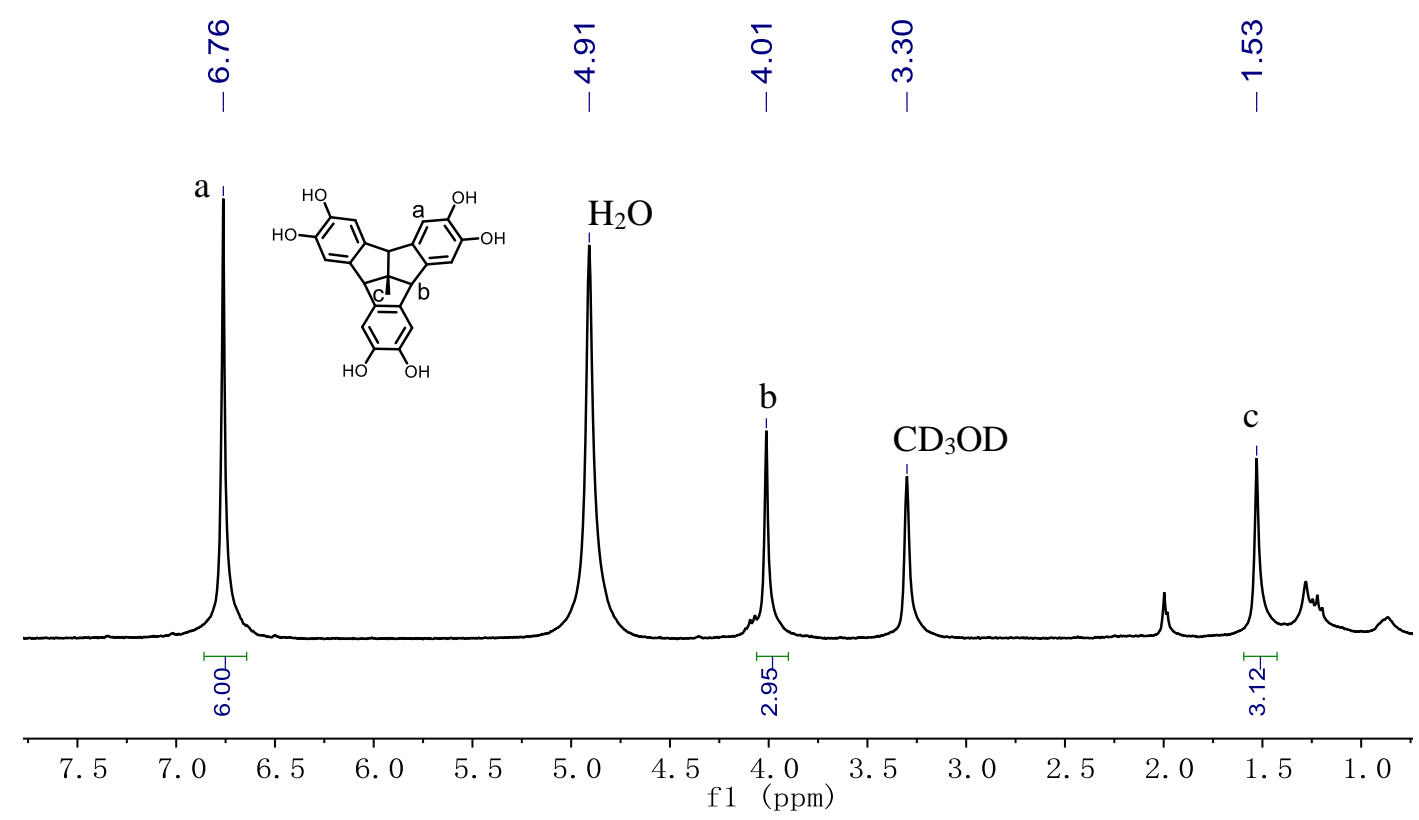

Figure S1. ${ }^{1} \mathrm{H}$ NMR spectra of TBTQ 


\subsection{Synthesis and activation of COFs}

\subsubsection{Synthesis and activation of COF-NUST-1 and COF-NUST-2}

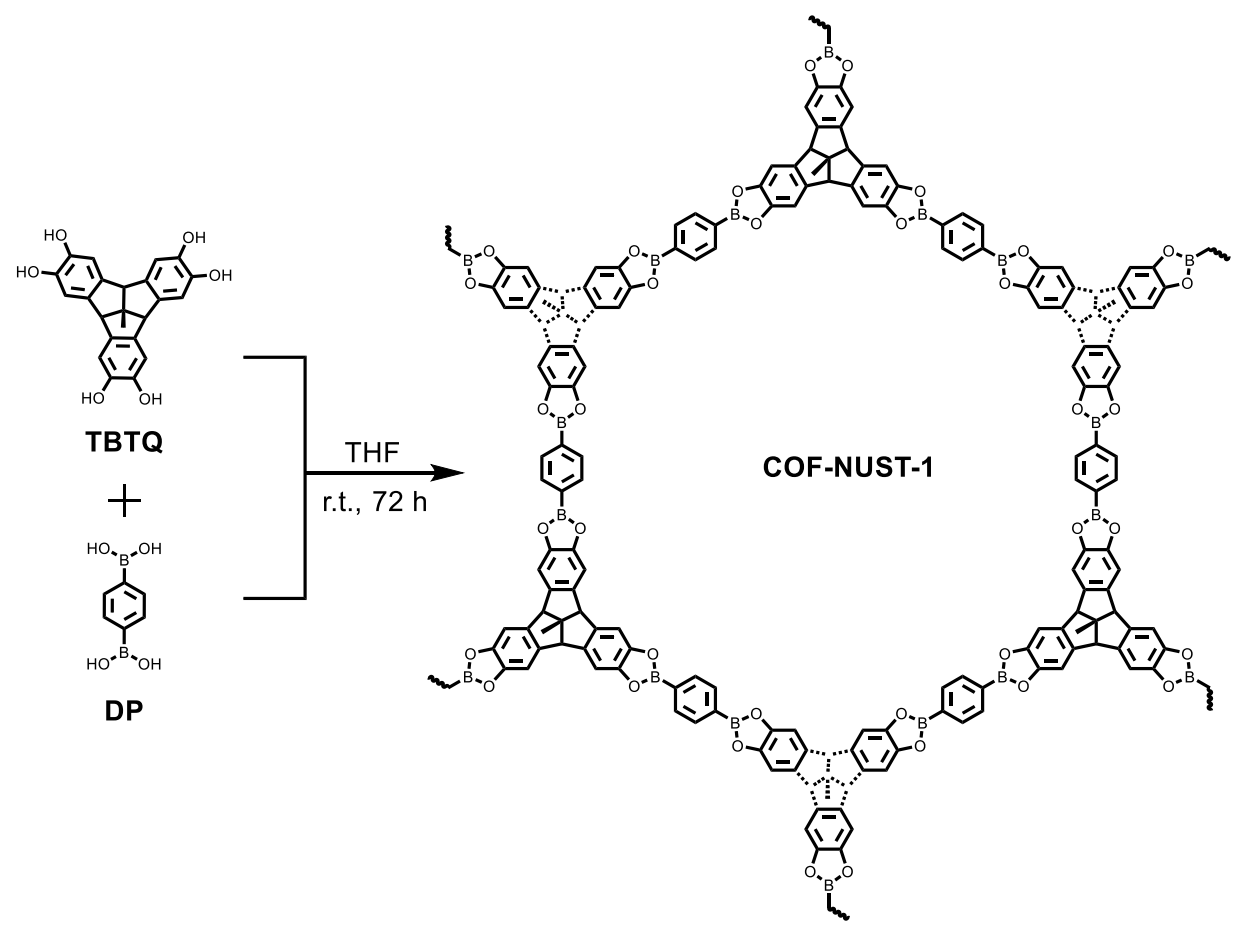

TBTQ (39.0 mg, $0.1 \mathrm{mmol})$, 1,4-phenylenebisboronic acid $(24.8 \mathrm{mg}, 0.15 \mathrm{mmol})$, anhydrous THF ( $4 \mathrm{~mL}$ ) were charged into a glass ampoule, the glass ampoule was flash frozen at $77 \mathrm{~K}$ using the liquid nitrogen bath and degassed by freeze-pump-thaw three times, and then was sealed. The glass ampoule was placed at room temperature for 3 days. Purple solid at the bottom of the glass ampoule was isolated by filtration and washed with anhydrous acetone, yielding purple powder COF-NUST-1. (Yield: $30 \mathrm{mg}, 60 \%$ ). 


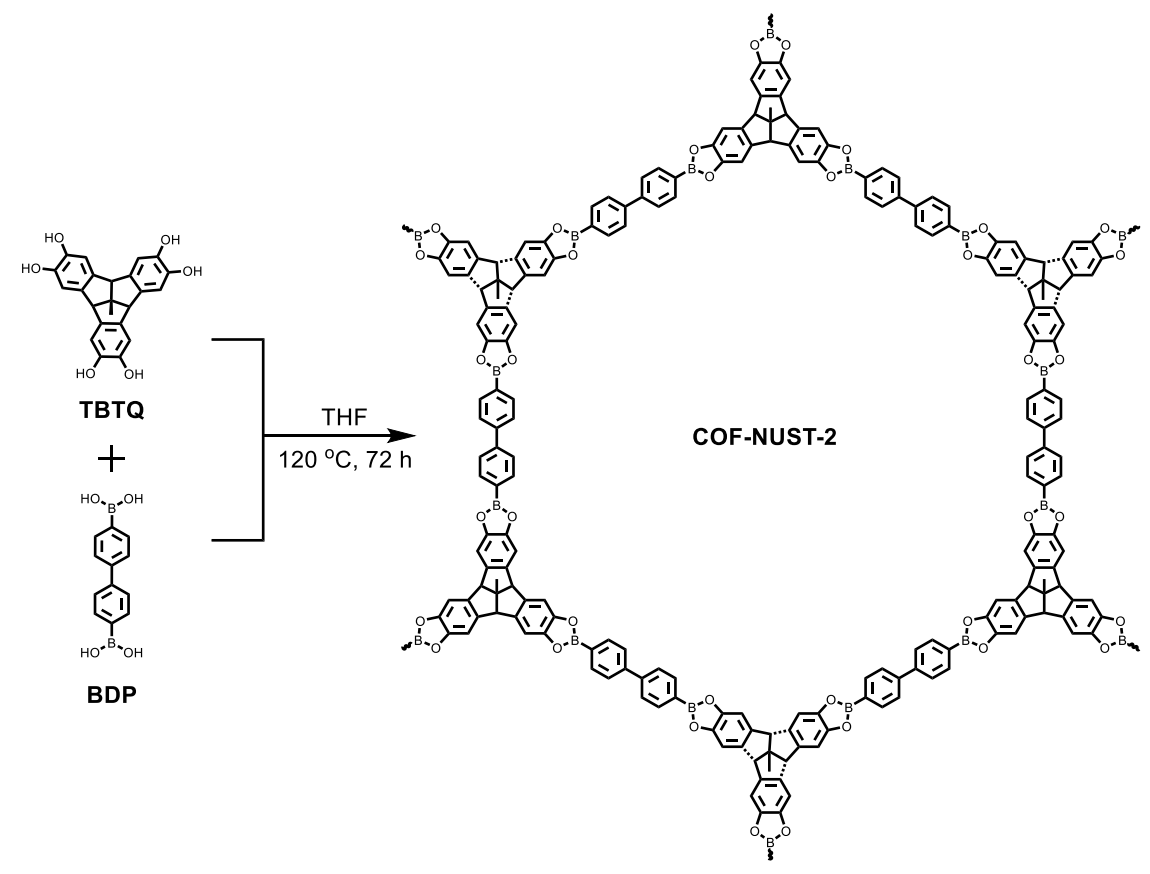

The COF-NUST-2 was prepared by solvothermal reactions in THF $(4.0 \mathrm{~mL})$ in the ampoule at $120{ }^{\circ} \mathrm{C}$. After three days, the reactions afforded sediment at the bottom of ampoule. Drying those solid at $50{ }^{\circ} \mathrm{C}$ under vacuum for $12 \mathrm{~h}$ yields COF-NUST-2 as a purple powder which was characterized by PXRD pattern and FTIR spectra.

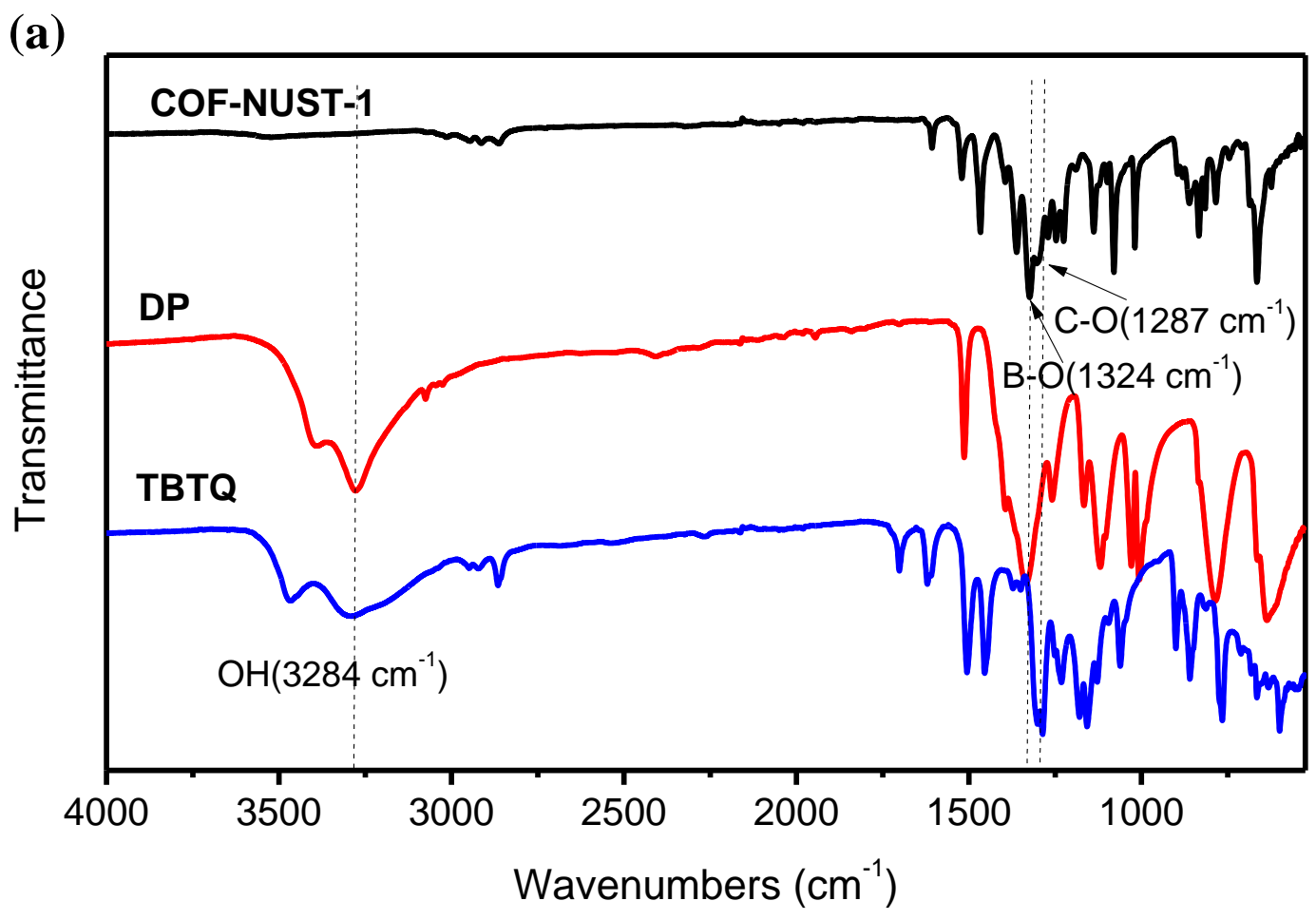


(b)

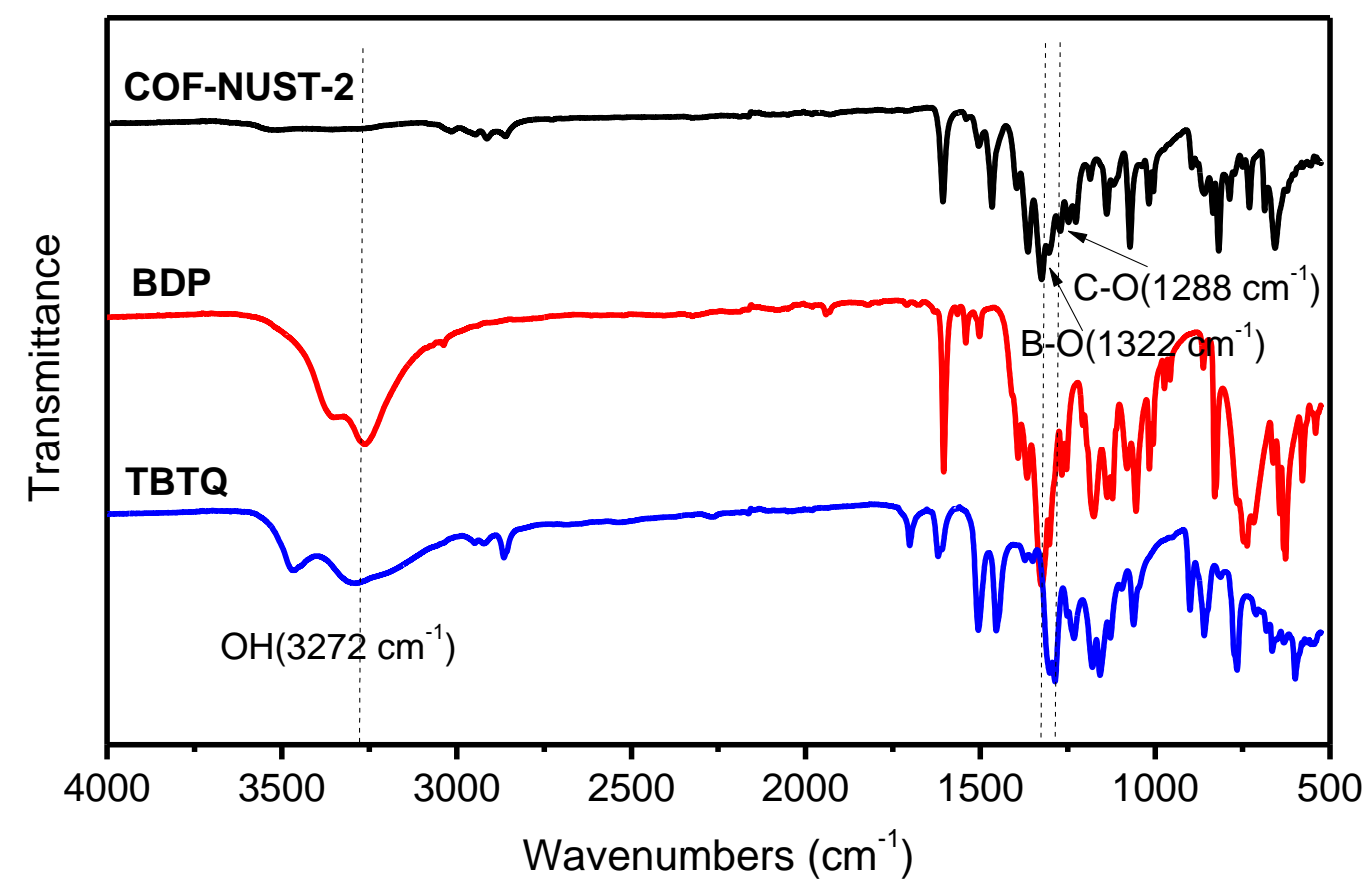

Figure S2. FT-IR spectra of (a) COF-NUST-1, DP, TBTQ; (b) COF-NUST-2, BDP, TBTQ

(a)

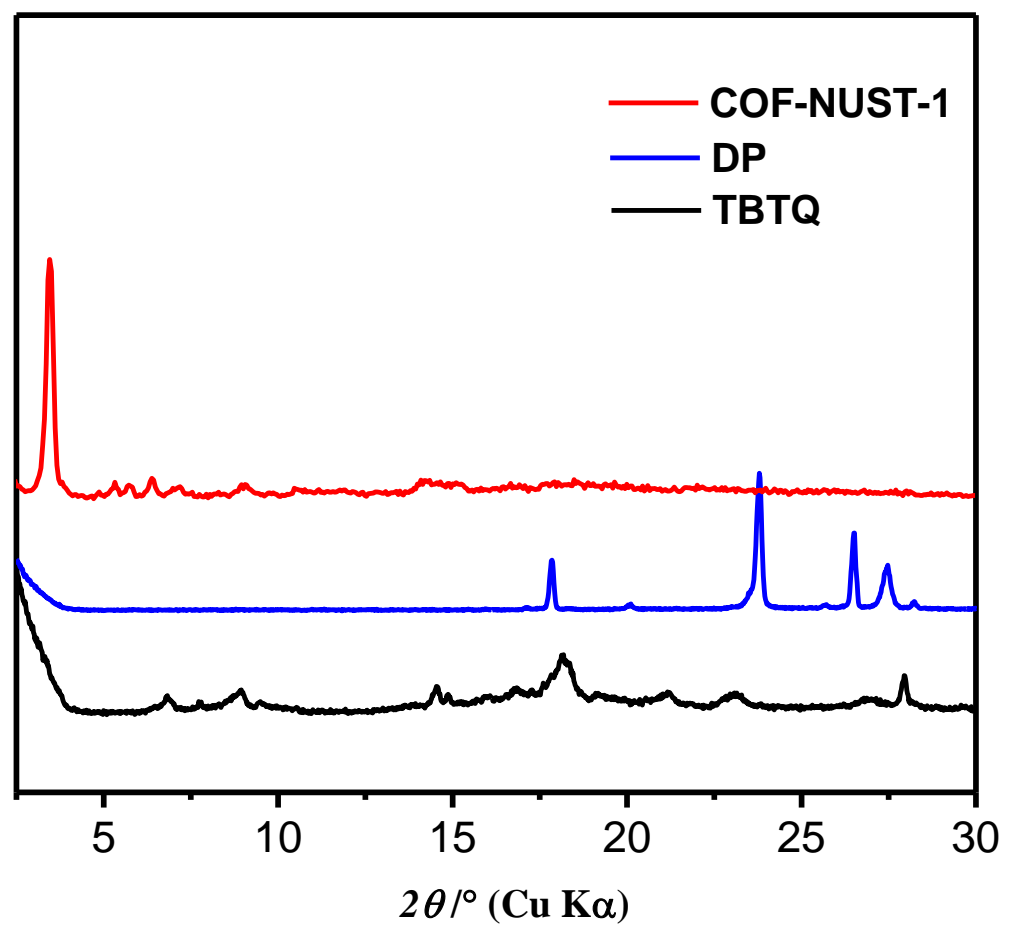


(b)

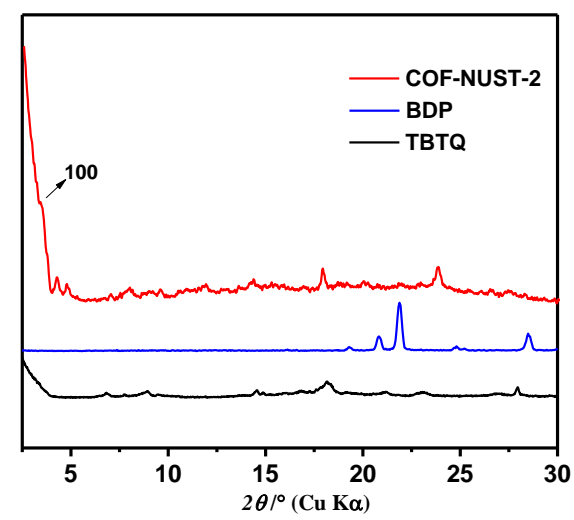

(c)

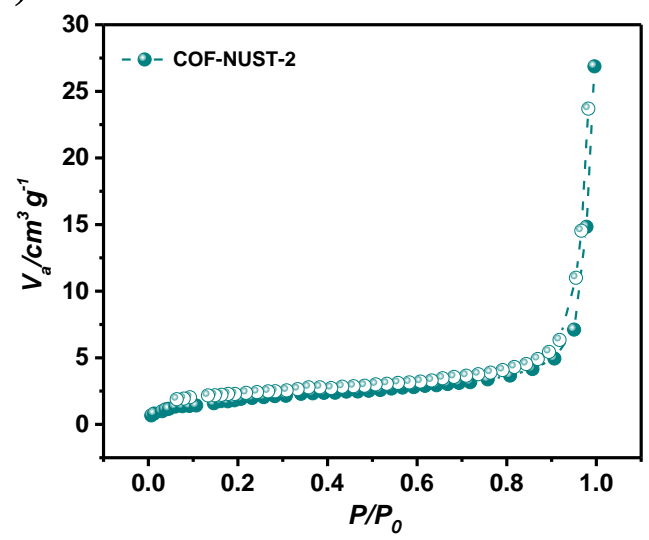

Figure S3. PXRD patterns of (a) COF-NUST-1, DP, TBTQ; (b) COF-NUST-2 and (c) Nitrogen sorption curves for COF-NUST-2

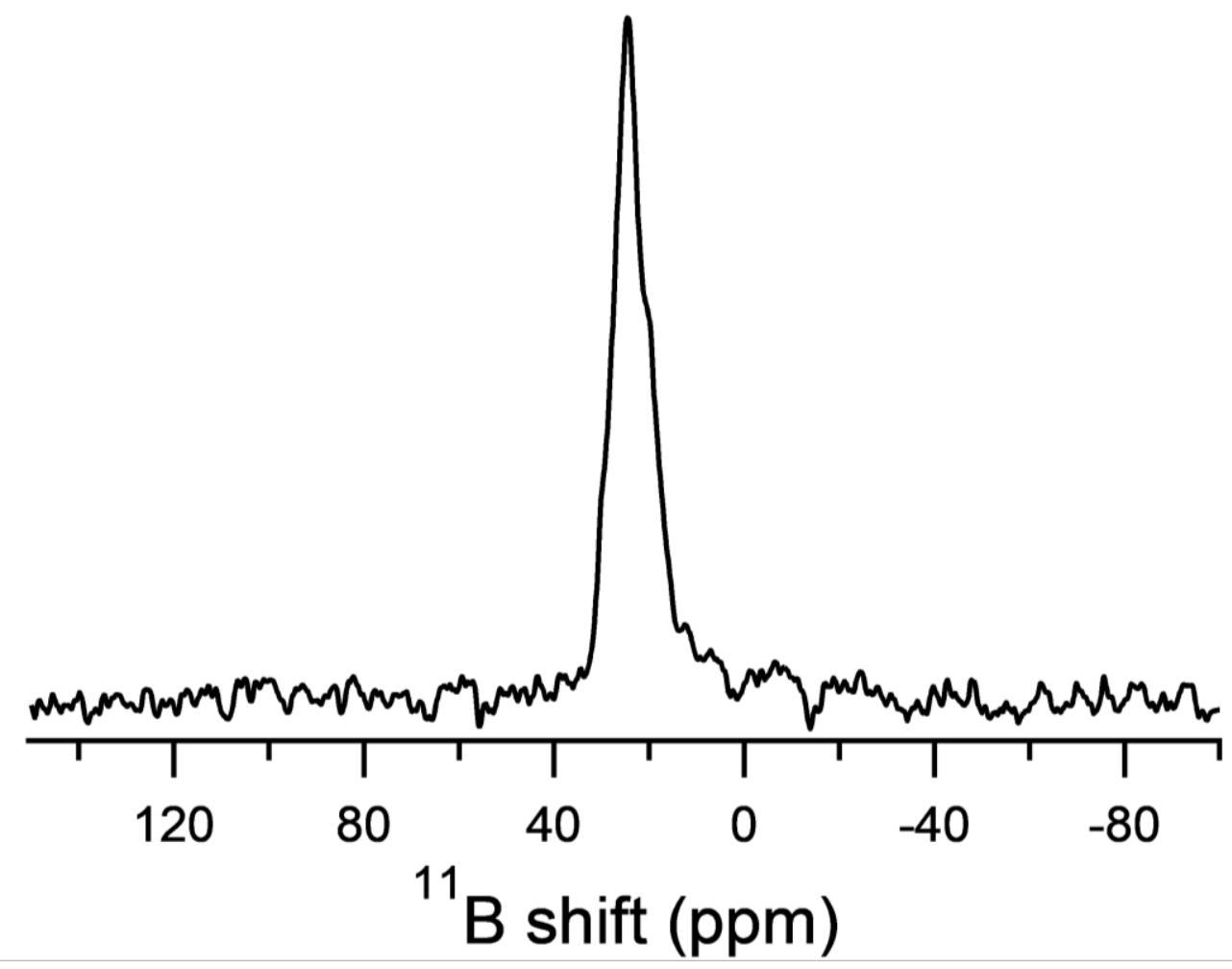

Figure S4. $1 \mathrm{D}^{11} \mathrm{~B}$ single pulse spectra of COF-NUST-1 


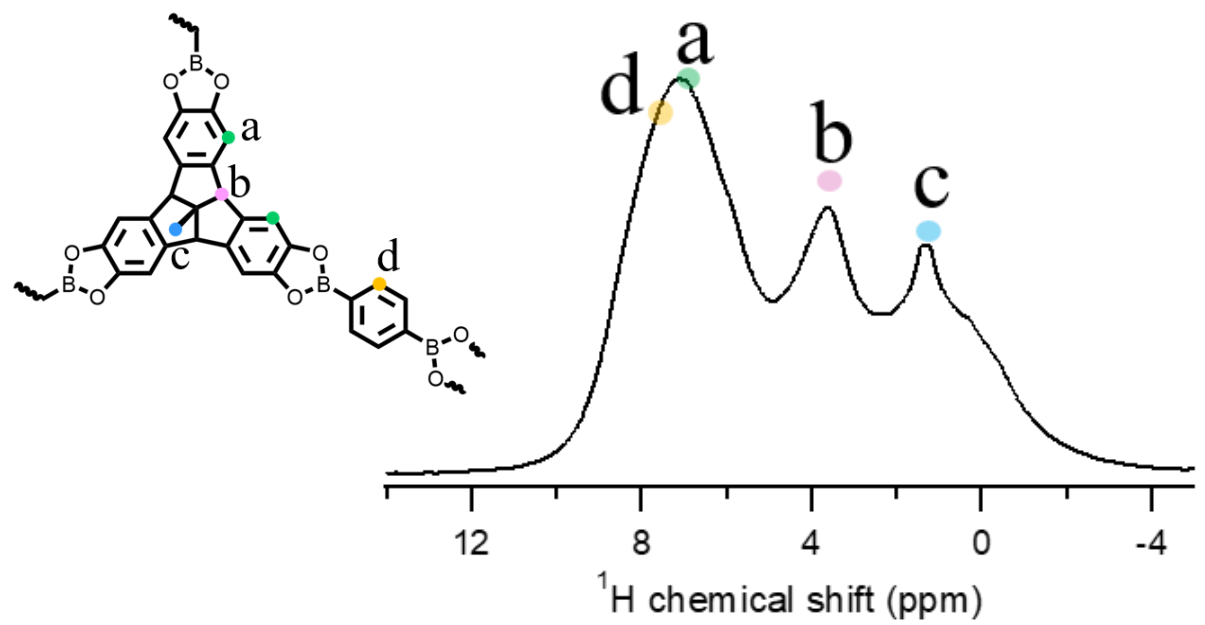

Figure S5. 1D ${ }^{1} \mathrm{H}$ single pulse spectra of COF-NUST-1

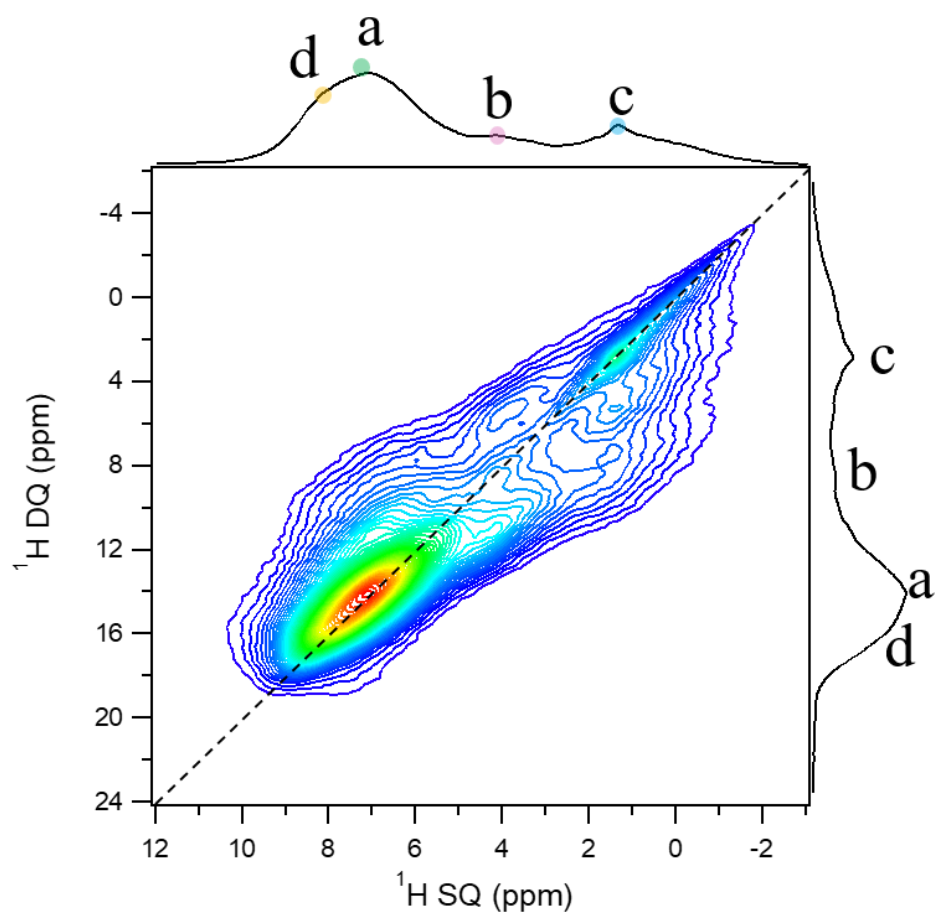

Figure S6. ${ }^{1} \mathrm{H}$ DQ/SQ spectra of COF-NUST-1 

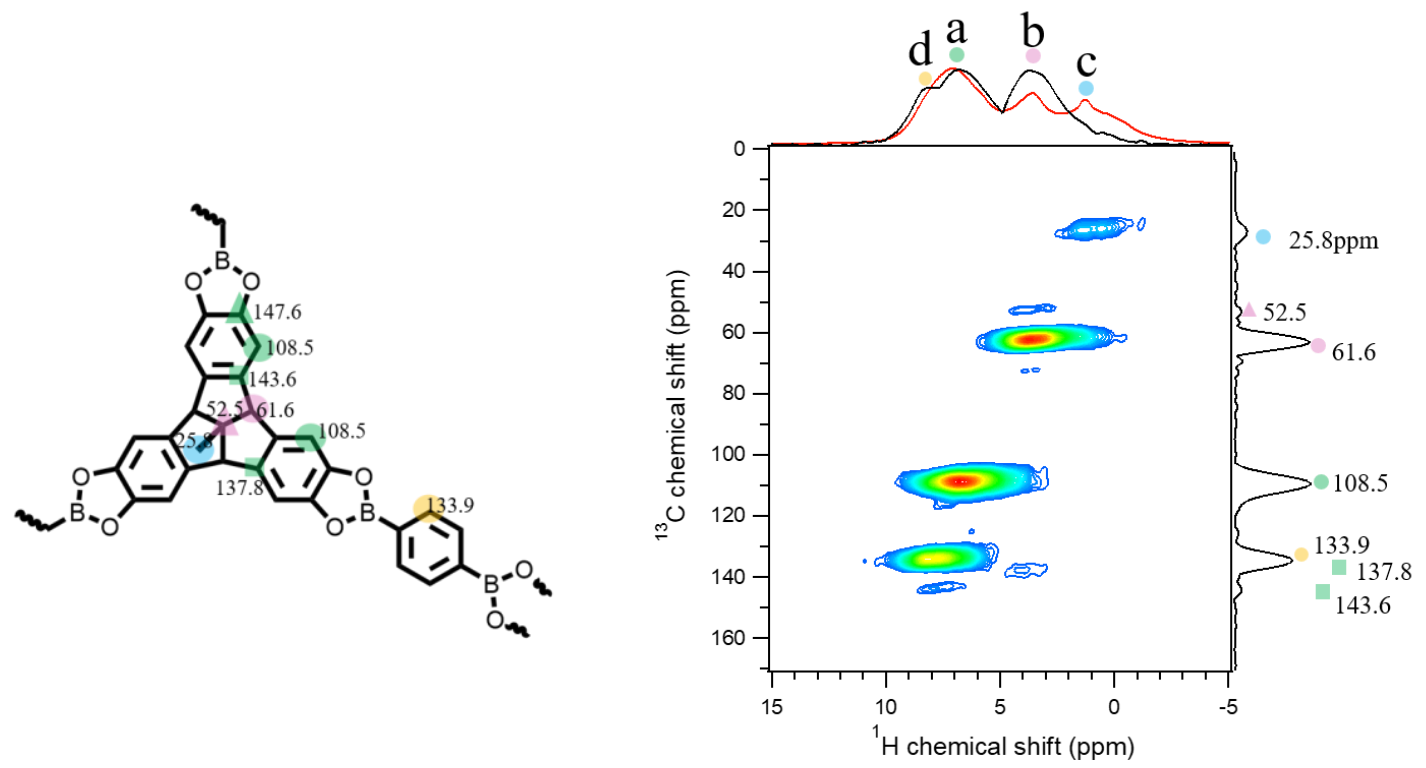

Figure S7. ${ }^{1} \mathrm{H}_{-}{ }^{13} \mathrm{C}$ HETCOR spectrum of COF-NUST-1, The HETCOR spectrum was measured with the contact time of $0.4 \mathrm{~ms}$.
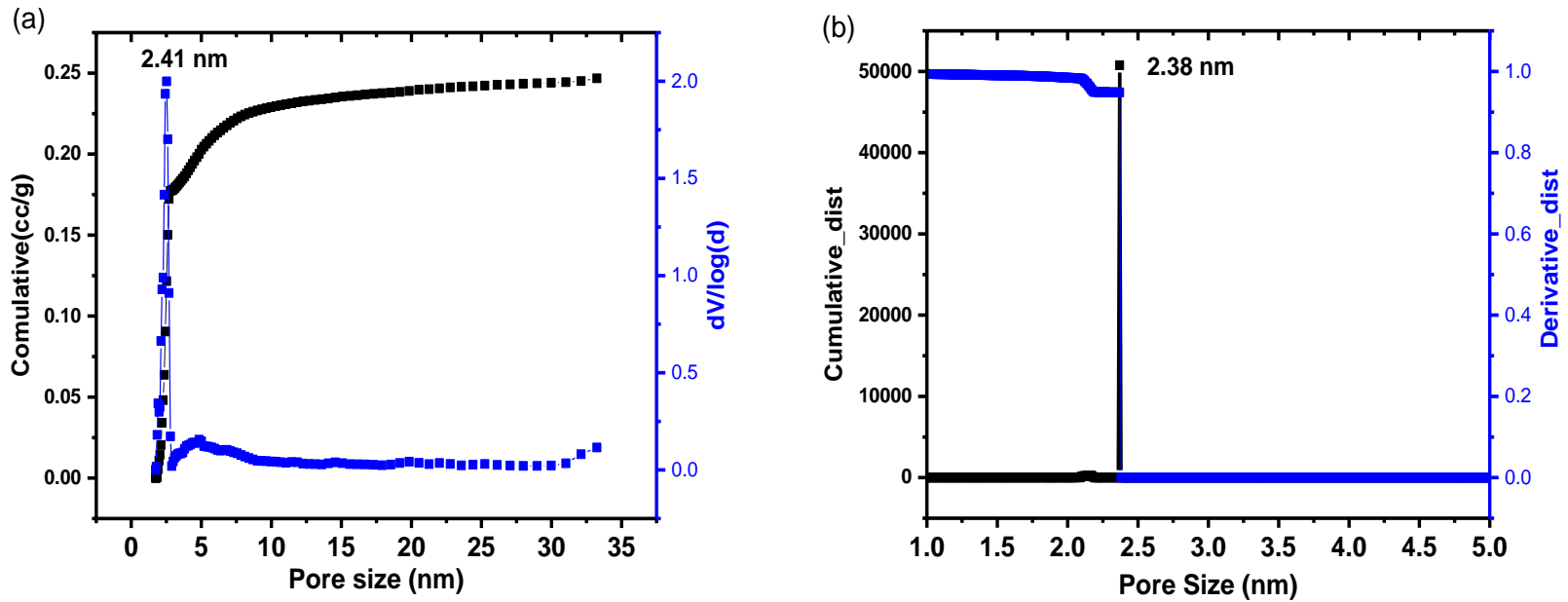

Figure S8. PSD of COF-NUST-1 calculated from (a) $\mathrm{N}_{2}$ sorption (slit/cylinder, QSDFT adsorption branch, fitting error: $0.243 \%$ ) (b) Zeo++ (probe_radius $=1.86 \AA$, $\mathrm{N}_{2}$ ).

\subsubsection{Structural modeling and Le Bail refinement fitting for COF-NUST-1}

Crystal structural modeling of COFs were generated using AuToGraFS ${ }^{\mathrm{S} 2}$ and optimized by the Density Functional Tight-Binding method $(\mathrm{SCC}-\mathrm{DFTB})^{\mathrm{S} 3}$. Powder indexing (TREOR) and Le Bail fitting were performed by EXPO $2014^{\mathrm{S} 4}$ and FullProf ${ }^{\mathrm{S} 5}$, Pore size distribution (PSD) were calculated from $\mathrm{N}_{2}$ sorption curves (experimental data) and Zeo++ open source software ${ }^{\mathrm{S} 6}$ (simulated crystal structure). 
a)
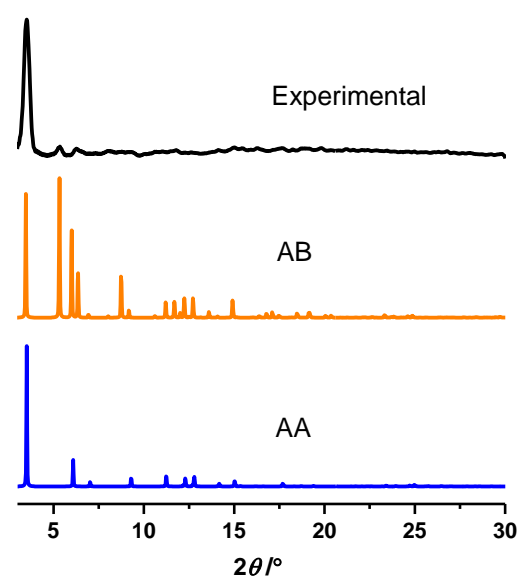

b)

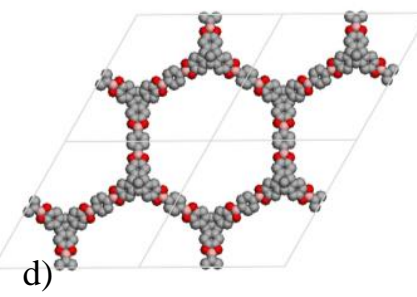

c)

e)
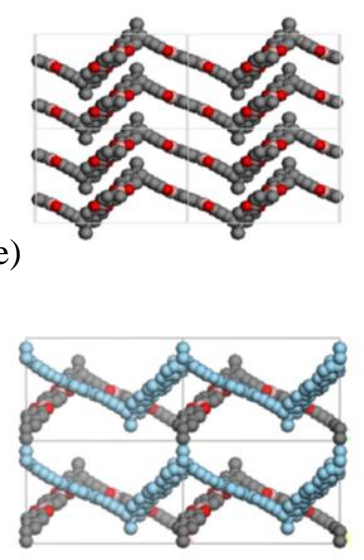

Figure S9. (a) Simulated PXRD patterns for eclipsed AA structure and staggered AB structure of COF-NUST-1. (b) Top and (c) side views of eclipsed structure. (d) Top and (e) side views of staggered structure for COF-NUST-1

Table S1. Fractional atomic coordinates for simulated COF-NUST-1.

\begin{tabular}{|l|l|l|l|}
\hline \multicolumn{3}{|c|}{ Space group: $P-3 M 1$} & \multicolumn{1}{l|}{$\mathrm{z}$} \\
\hline 3D hexagonal; $\mathrm{a}=\mathrm{b}=29.15 \AA, \mathrm{c}=16.56 \AA ; \alpha=\beta=90^{\circ}, \gamma=120^{\circ}$ & $\mathrm{y}$ & 0.46025 \\
\hline Atom & \multicolumn{1}{|c|}{$\mathrm{x}$} & 0.60609 & 0.42238 \\
\hline C1 & 0.34576 & 0.56055 & 0.38645 \\
\hline C2 & 0.34134 & 0.56486 & 0.35077 \\
\hline C3 & 0.38706 & 0.60716 & 0.26807 \\
\hline O4 & 0.47368 & 0.47099 & 0.23115 \\
\hline C5 & 0.44565 & 0.44588 & 0.03976 \\
\hline C6 & 0.47077 & 0.34582 & 0.0774 \\
\hline C7 & 0.60598 & 0.34145 & 0.11314 \\
\hline C8 & 0.5604 & 0.38722 & 0.14866 \\
\hline C9 & 0.5647 & 0.39303 & 0.42259 \\
\hline O10 & 0.52614 & 0.52524 & 0.28104 \\
\hline H11 & 0.3066 & 0.44898 & 0.21818 \\
\hline H12 & 0.4061 & 0.40633 & 0.07719 \\
\hline H13 & 0.44876 & 0.30671 & 1.15992 \\
\hline H14 & 0.5251 & 1.6464 & 0.17206 \\
\hline H15 & 1.3536 & 0.44813 & 0.32728 \\
\hline B16 & 0.55187 & 0.55207 & 0.28711 \\
\hline B17 & 0.44793 & 0.52559 & 0.21213 \\
\hline C18 & 0.47441 & 0.47463 & -0.00689 \\
\hline C19 & 0.52537 & 0.3908 & \\
\hline C20 & -0.3046 & & \\
\hline
\end{tabular}




\begin{tabular}{|l|l|l|l|}
\hline $\mathrm{H} 21$ & -0.28945 & 0.42109 & -0.05416 \\
\hline $\mathrm{C} 22$ & 0.30467 & 0.60935 & 0.50717 \\
\hline $\mathrm{H} 23$ & 0.28907 & 0.57814 & 0.55289 \\
\hline $\mathrm{C} 24$ & -0.33333 & 0.33333 & -0.04248 \\
\hline $\mathrm{C} 25$ & -0.33333 & 0.33333 & -0.13683 \\
\hline $\mathrm{C} 26$ & -0.33333 & 0.33333 & 0.45557 \\
\hline $\mathrm{C} 27$ & -0.33333 & 0.33333 & 0.36003 \\
\hline $\mathrm{H} 28$ & 1.37326 & 1.68663 & 1.665 \\
\hline
\end{tabular}

\subsubsection{Synthesis of COF-LZU-1}

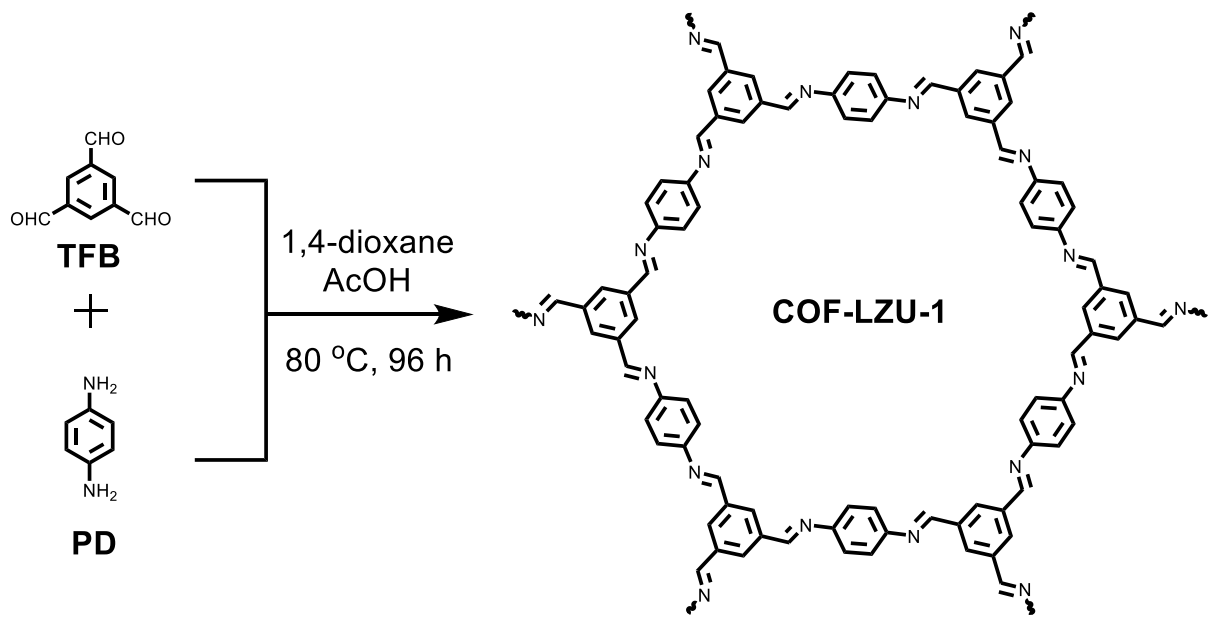

COF-LZU-1 was synthesized according to the reported literature with a modified procedure. ${ }^{\text {S7 }}$ Benzene-1,3,5-tricarbaldehyde (25.9 mg, $0.16 \mathrm{mmol}$ ) and 1,4-diaminobenzene (25.9 $\mathrm{mg}, 0.24 \mathrm{mmol})$ were weighed into a $30 \mathrm{ml}$ glass vial with 1,4 -dioxane $(6 \mathrm{~mL})$. The solution was heated to $80{ }^{\circ} \mathrm{C}$ for 3 minutes to ensure dissolution giving yellow clean solution and then cooled to room temperature. Distilled $\mathrm{H}_{2} \mathrm{O}(1.2 \mathrm{~mL})$ was added to the solution, followed by glacial acetic acid $(1.8 \mathrm{~mL})$. The resulting suspension was sealed and placed in an oven at $80{ }^{\circ} \mathrm{C}$ and left undisturbed for $96 \mathrm{~h}$, The COF yellow solid was isolated by centrifugation and washed with 1,4-dioxane $(3 \times 10 \mathrm{~mL})$ and then filtered. The sample was then transferred to vacuum chamber and evacuated to $20 \mathrm{mTorr}$ at $50{ }^{\circ} \mathrm{C}$ for $24 \mathrm{~h}$, yielding yellow powder COF-LZU-1 (Yield: $35 \mathrm{mg}, 82 \%$ ). 


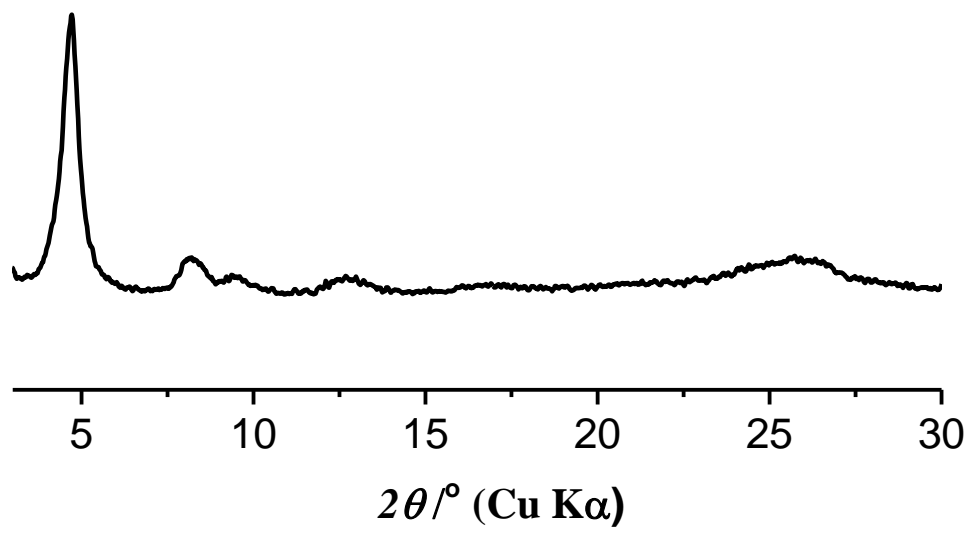

Figure S10. PXRD pattern of COF-LZU-1

\subsubsection{Synthesis and activation of COF-TFPA}

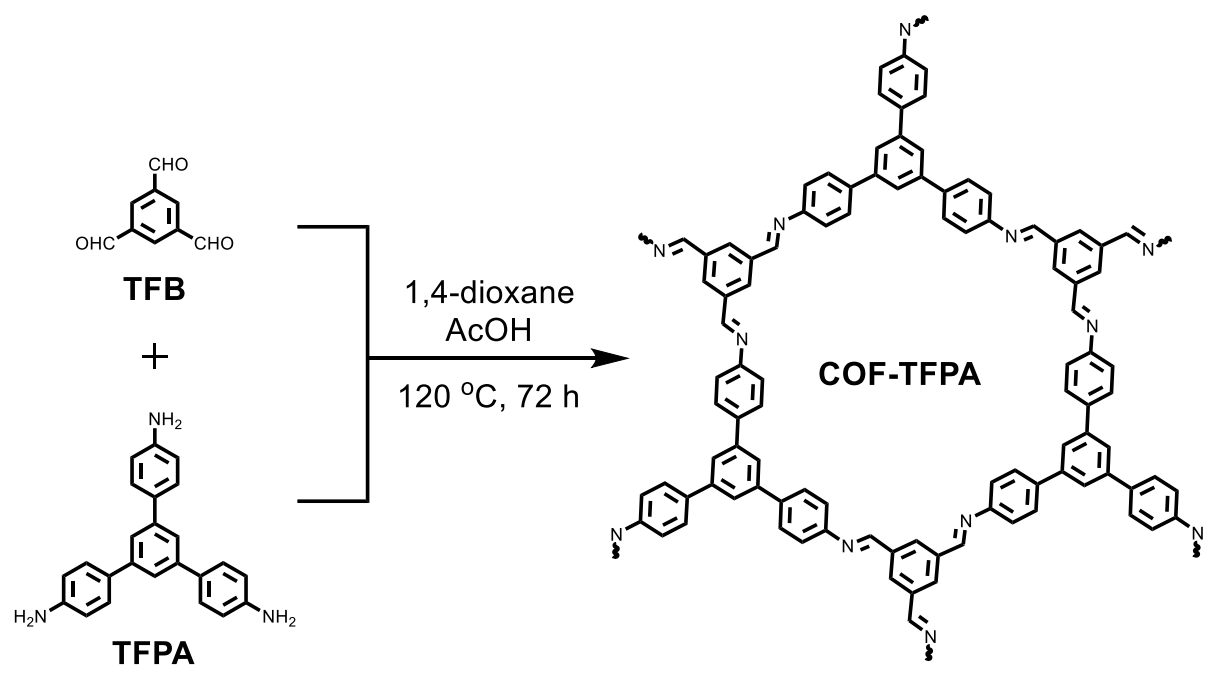

COF-TFPA was synthesized according to the reported literature with a modified procedure. ${ }^{\mathbf{S 8}}$ Benzene-1,3,5-tricarbaldehyde (25.9 $\mathrm{mg}, 0.16 \mathrm{mmol})$ and TFPA $(56.2 \mathrm{mg}, 0.16 \mathrm{mmol})$ were weighed into a glass ampoule with 1,4-dioxane $(3 \mathrm{~mL})$. The solution was ultrasound for 5 minutes to obtain yellow turbid solution. $3 \mathrm{M}$ acetic acid $(0.6 \mathrm{~mL})$ was pure into the glass ampoule as catalyst. The glass ampoule was flash frozen at $77 \mathrm{~K}$ using the liquid nitrogen bath and degassed by freeze-pump-thaw three times, and then was sealed. The glass ampoule was placed at $120{ }^{\circ} \mathrm{C}$ for 3 days. The yellow solid was isolated by centrifugation and washed with 1,4-dioxane $(3 \times 10 \mathrm{~mL})$ and then filtered. The sample was then transferred to vacuum chamber and evacuated to 20 mTorr at 
$50{ }^{\circ} \mathrm{C}$ for $24 \mathrm{~h}$, yielding yellow powder COF-TFPA (Yield: $63 \mathrm{mg}, 90 \%$ ).

\section{COF-TFPA}

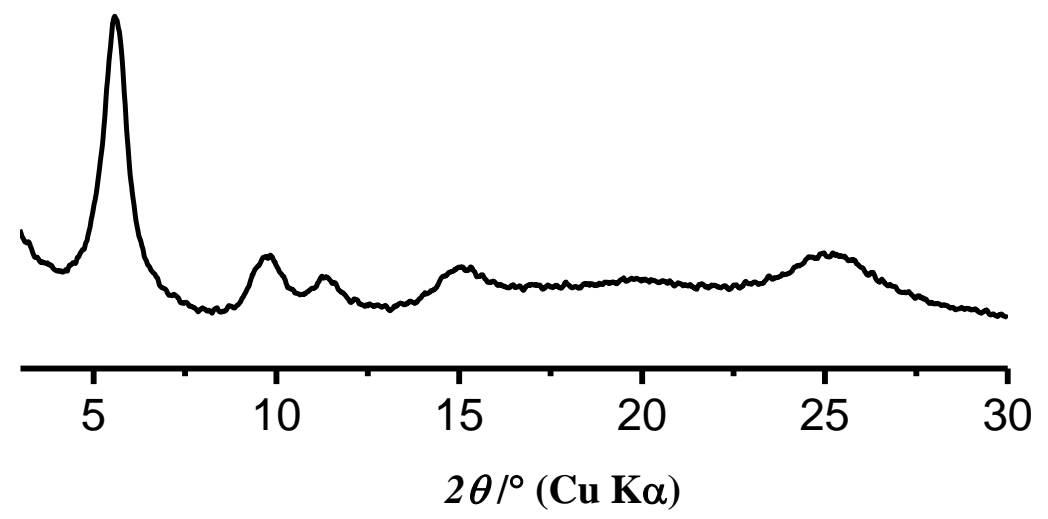

Figure S11. PXRD pattern of COF-TFPA

\subsection{Synthesis and characterization of Cages}

\subsubsection{Synthesis of Cage-NUST-1}

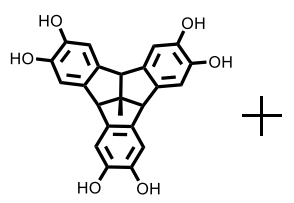

TBTQ

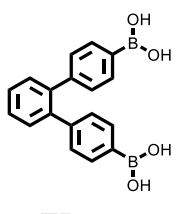

TP

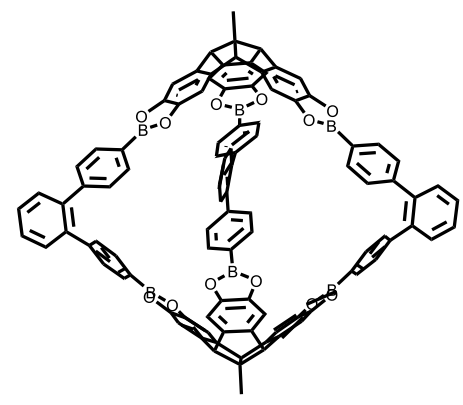

Cage-NUST-1

TBTQ (39.0 mg, $0.1 \mathrm{mmol}, 1 \mathrm{eq})$ and ortho-phenylene diboronic acid (47.7 mg, $0.15 \mathrm{mmol}$, 1.5 eq) were dissolved in $4 \mathrm{~mL}$ anhydrous THF and molecular sieves $4 \AA$ were added. After three days, the solvent was removed under reduced pressure. The residue was solved in $\mathrm{CHCl}_{3}$ and unsolved material was filtered off. The solvent was removed under reduced pressure to give Cage-NUST-1 as a bluish violet solid $(50.1 \mathrm{mg}, 66 \%) .{ }^{1} \mathrm{H}-\mathrm{NMR}\left(500 \mathrm{MHz}, \mathrm{CDCl}_{3}\right): \delta 7.84(\mathrm{~d}, 12 \mathrm{H}$, Ha), 7.46-7.39 (m, 12H), 7.27 (d, 12H), $7.24(\mathrm{~s}, 12 \mathrm{H}), 4.42$ (s, 6H), 1.43 (s, 6H). 


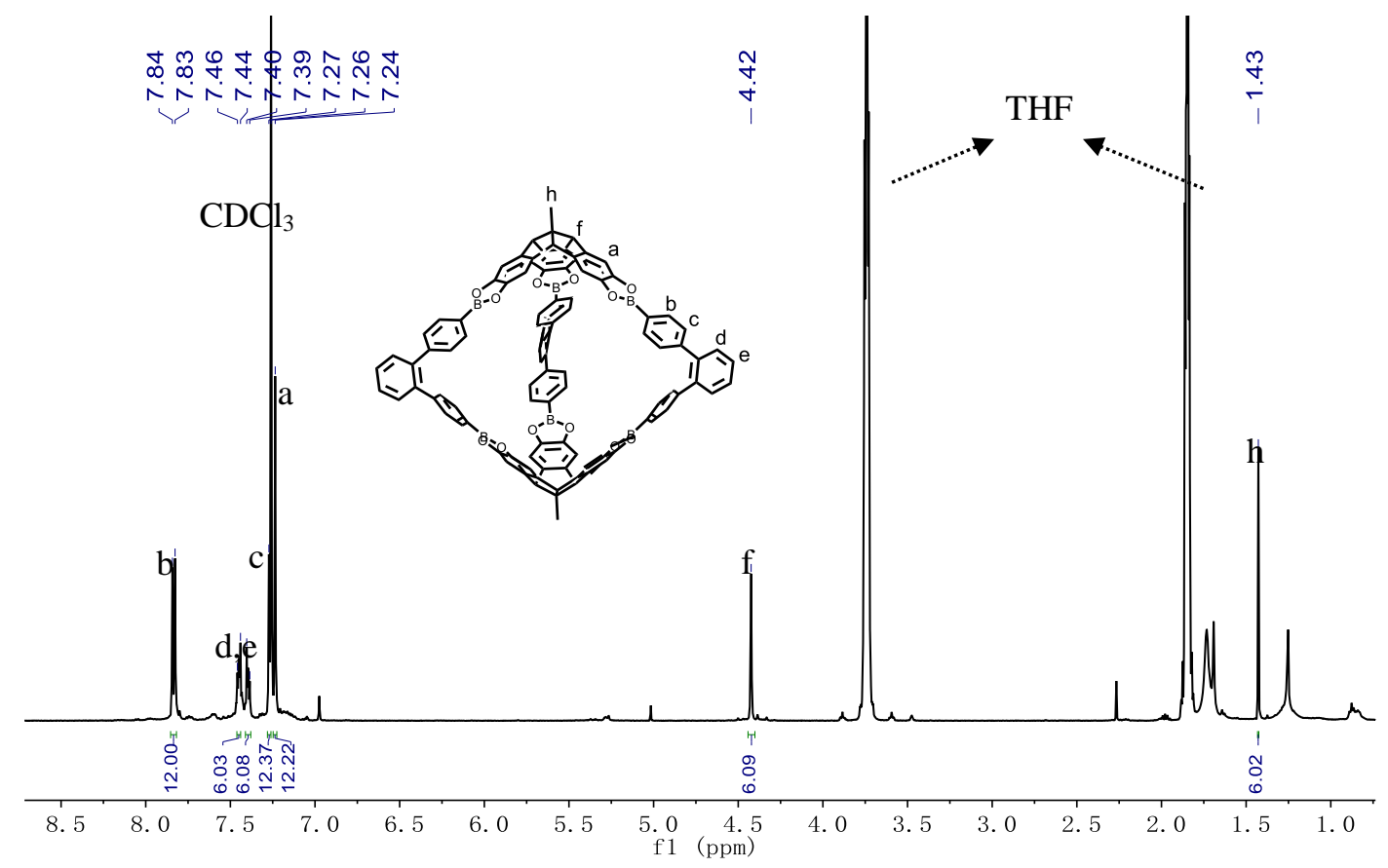

Figure S12. ${ }^{1} \mathrm{H}$ NMR spectra of Cage-NUST-1

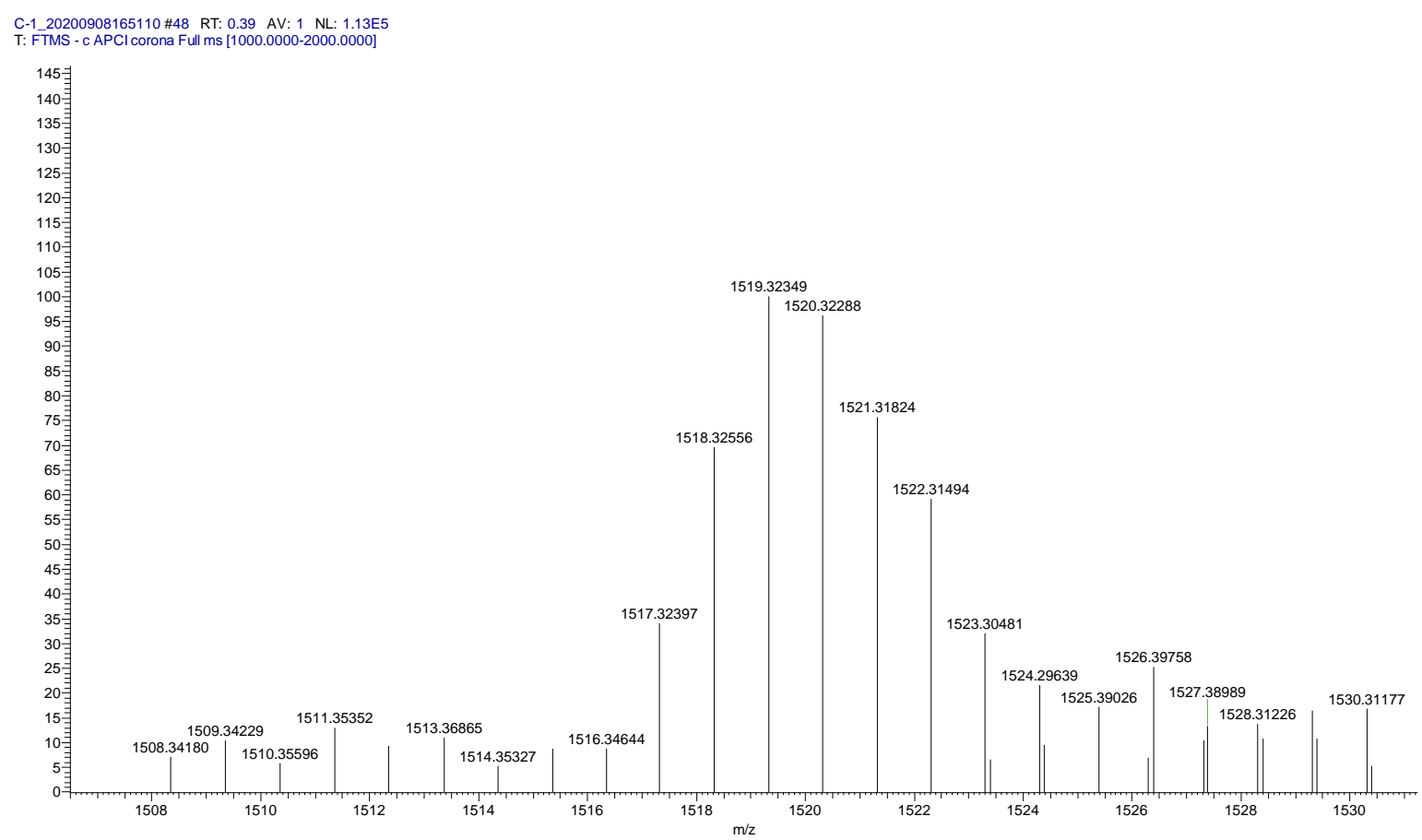

Figure S13. APCI-MS spectra of Cage-NUST-1 


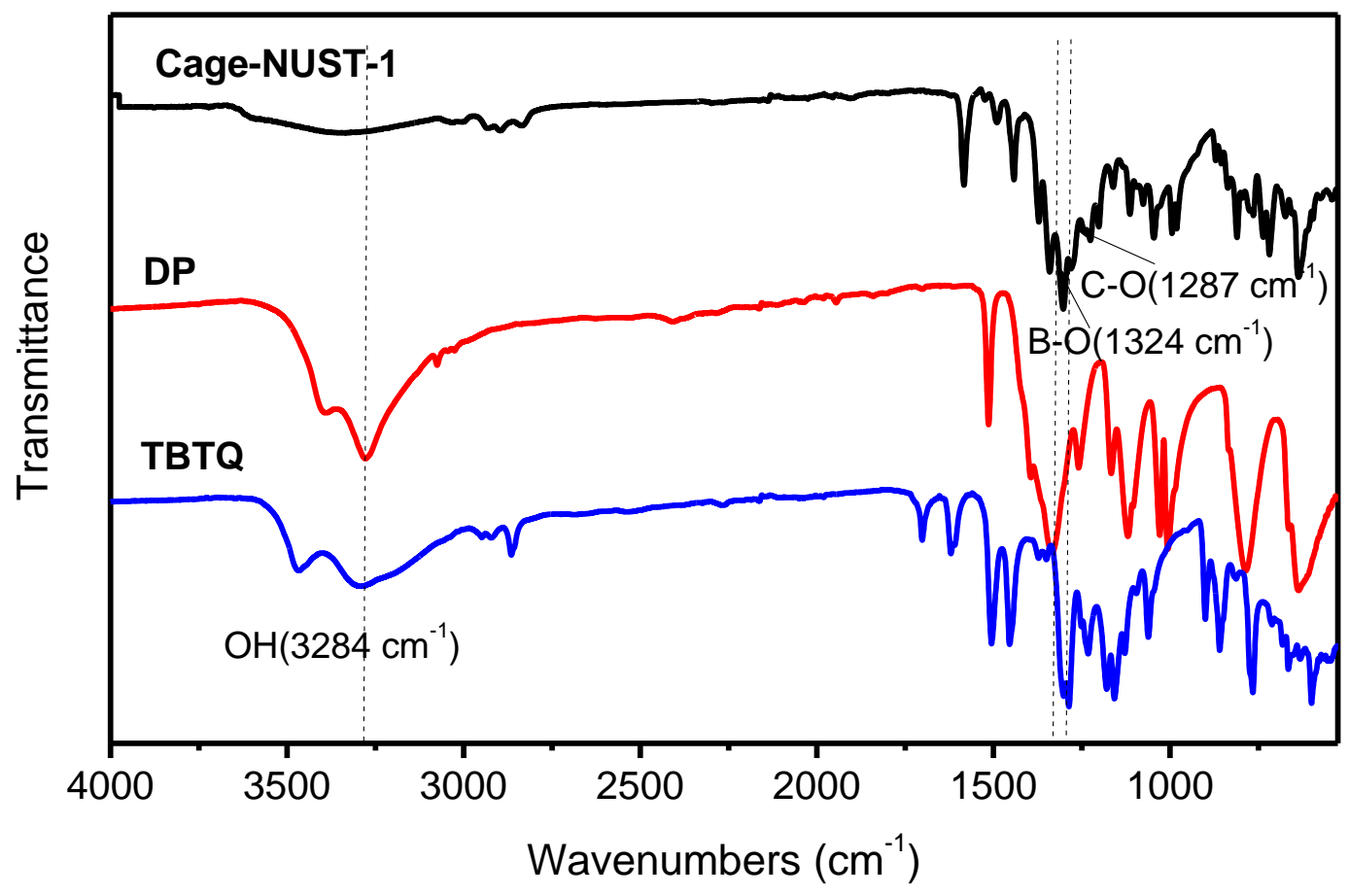

Figure S14. FT-IR spectra of Cage-NUST-1, DP, TBTQ

\subsubsection{Synthesis of Cage 1}

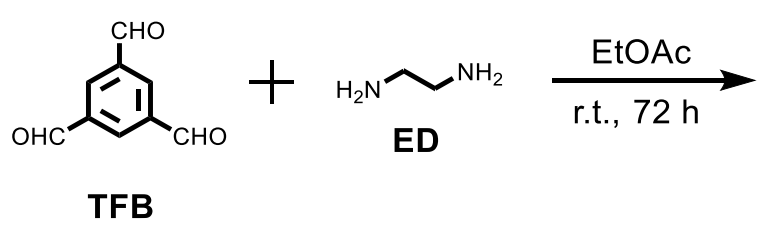

TFB

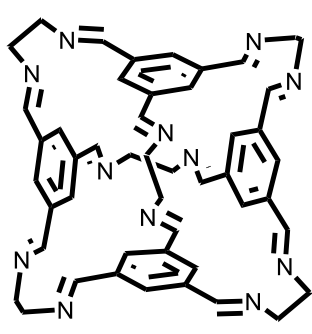

Cage 1

Cage 1 was synthesized according to the reported literature. ${ }^{59} 1,3,5$-triformylbenzene $(50 \mathrm{mg}$, $0.31 \mathrm{mmol})$ was dissolved into a glass vial with Ethyl acetate $(35 \mathrm{~mL})$ at room temperature. Then a solution of ethylene diamine $(31 \mu \mathrm{L}, 0.47 \mathrm{mmol})$ in EtOAc $(5 \mathrm{~mL})$ was added. After 30 minutes, a turbid solution was observed to form. The resulting mixture was left covered for $72 \mathrm{~h}$ without stirring. Finally, pale white needles-like crystals were observed in the solution. The crystals were harvested carefully by getting rid of the excess solvent, washed with ethyl acetate, and dried to give Cage 1 (58 mg, 74\%). ${ }^{1} \mathrm{H}$ NMR $\left(\mathrm{CDCl}_{3}, 500 \mathrm{MHz}\right) \delta 8.19(\mathrm{~s}, 12 \mathrm{H}), 7.93(\mathrm{~s}, 12 \mathrm{H}), 4.03(\mathrm{~s}, 24 \mathrm{H})$. The spectral data are in accordance with those in the literature reports. 


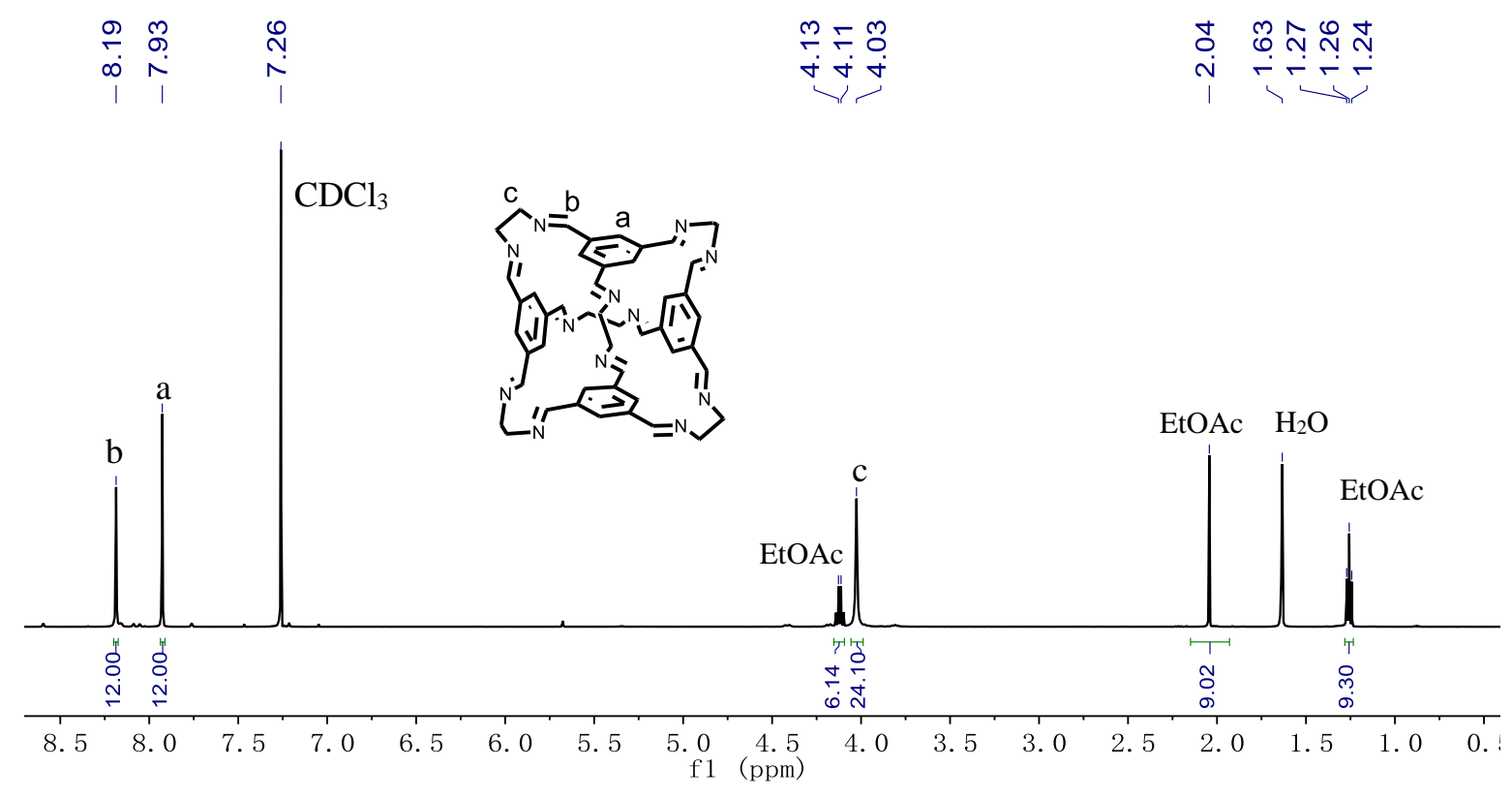

Figure S15. ${ }^{1} \mathrm{H}$ NMR spectra of Cage 1

\subsection{Transformation between COFs and Cages}

\subsubsection{The transformation from COF-NUST-1/2 to Cage-NUST-1}

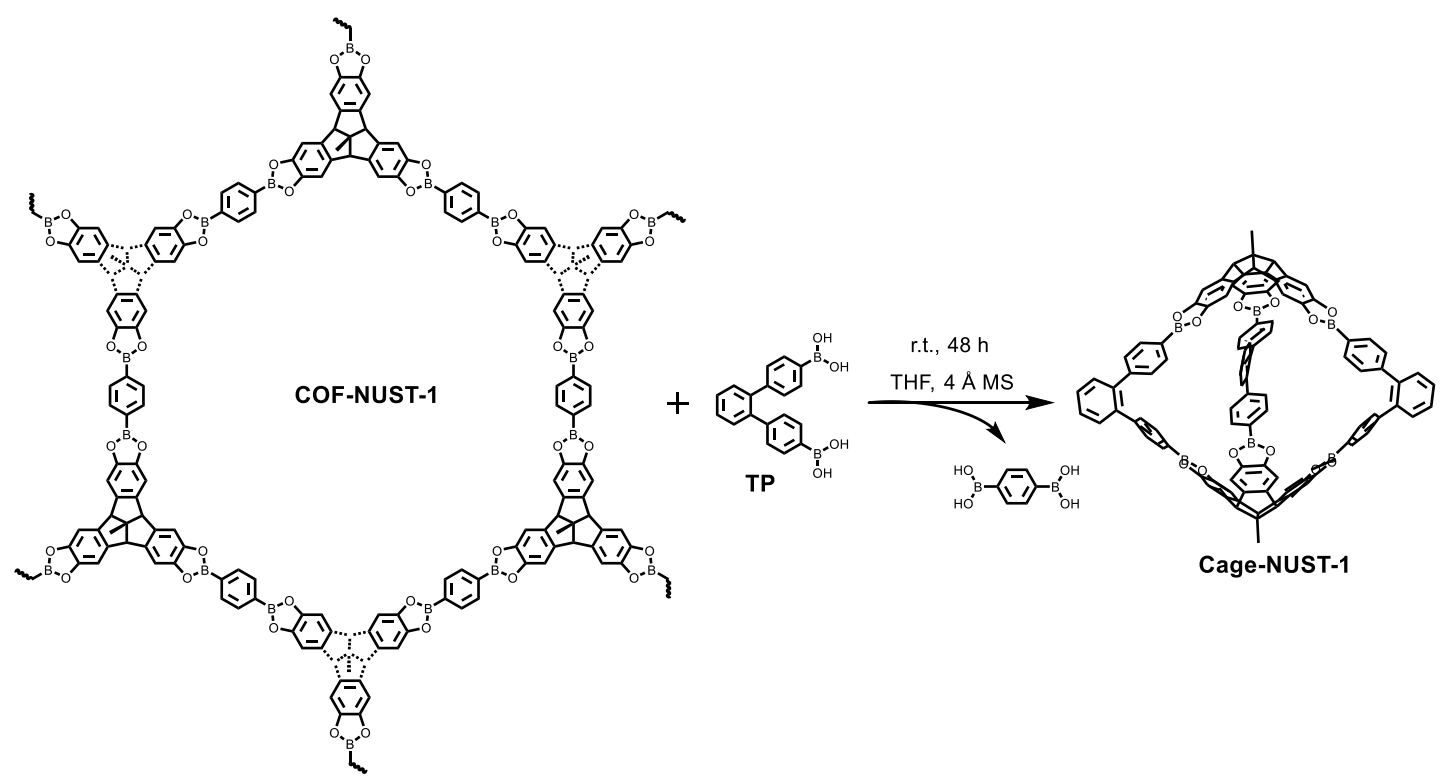

COF-NUST-1 (20 mg) was weighed into a $20 \mathrm{ml}$ glass vial, anhydrous THF (4 mL) was added into the glass vial. The molecular sieve $4 \AA$ was added after the addition of ortho-phenylene diboronic acid (23.4 $\mathrm{mg}$ ) with a 2:3 molecular ratio. A turbidity solution was observed to form within 30 min after ortho-phenylene diboronic acid addition to the glass vial. The solution gradually 
turns dark blue after $48 \mathrm{~h}$. The solvent was removed under reduced pressure, and the residue was solved in $\mathrm{CHCl}_{3}$ and unsolved material was filtered off. The solvent was removed under reduced pressure to give Cage-NUST-1 as a mauve solid $(50.1 \mathrm{mg}, 66 \%)$. The transformation from COF-NUST-2 to Cage-NUST-1 was also proceeded in a similar way. ${ }^{1} \mathrm{H}$ NMR spectra indicated that the transformation can be successfully implemented (Figure S17b).

The experiment based on TP monomers' concentration has been accomplished, and the result shows that the transformation from COF-NUST-1 to Cage-NUST-1 could still be realized which was characterized by ${ }^{1} \mathrm{H}$ NMR spectra (Figure S17c).

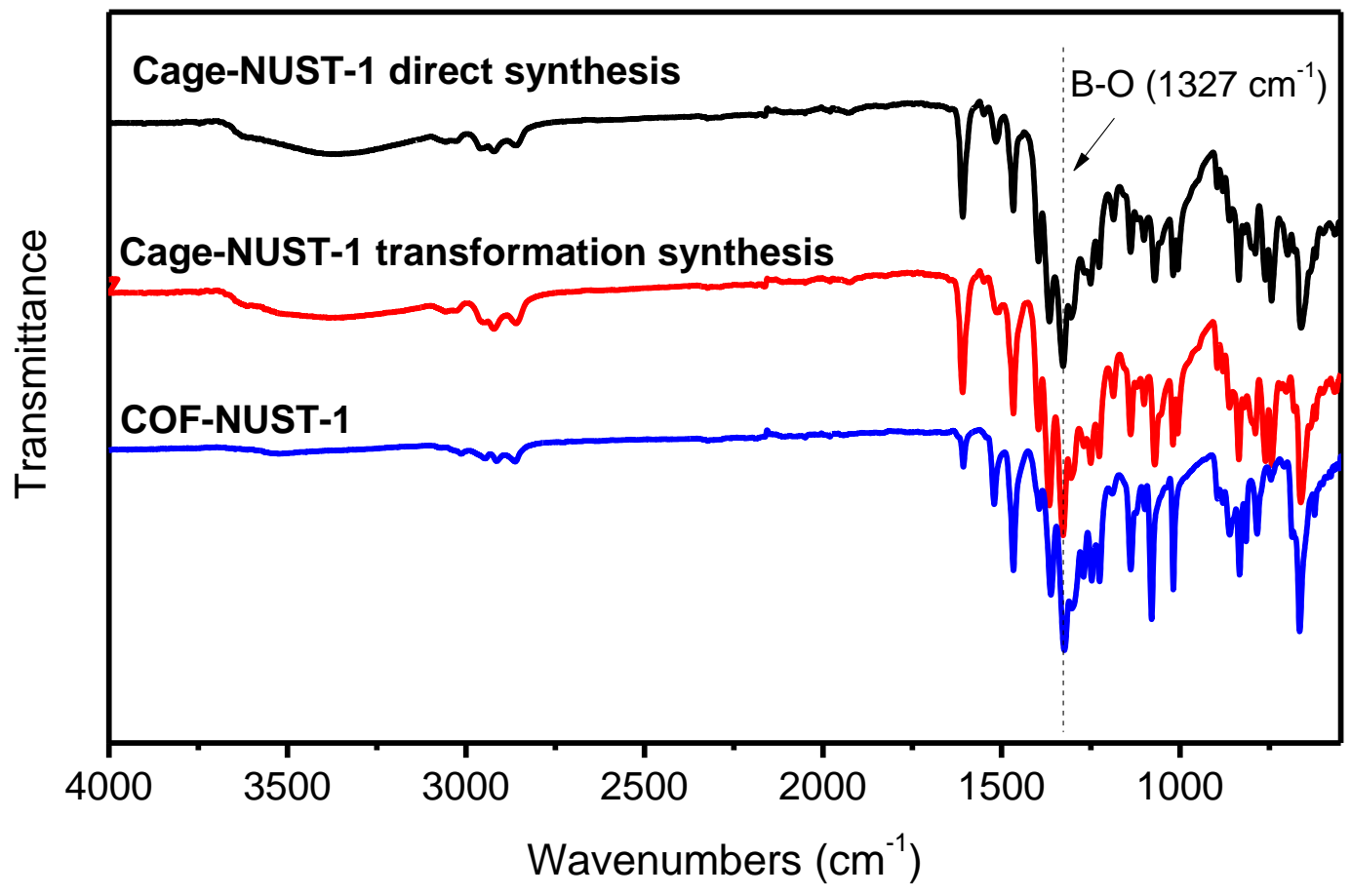

Figure S16. FT-IR spectra of COF-NUST-1, Cage-NUST-1. 


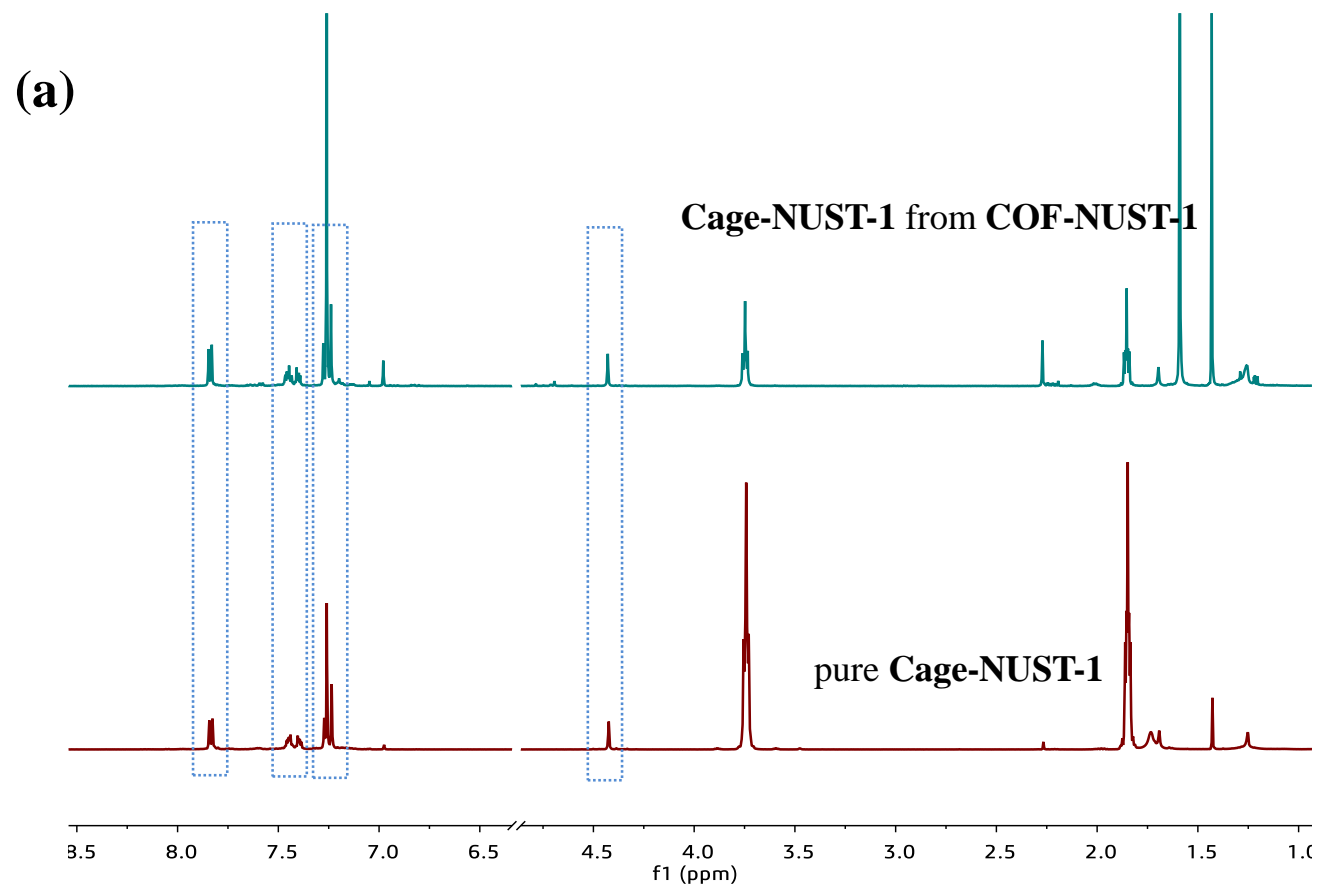

(b)

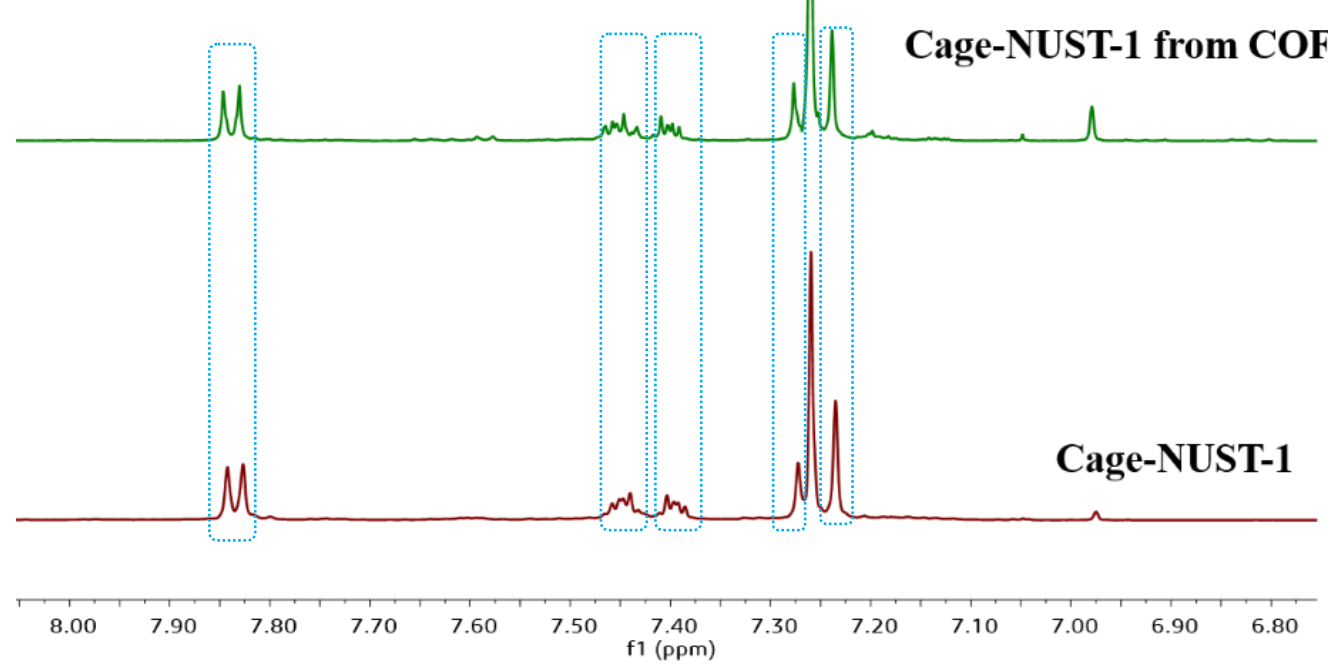




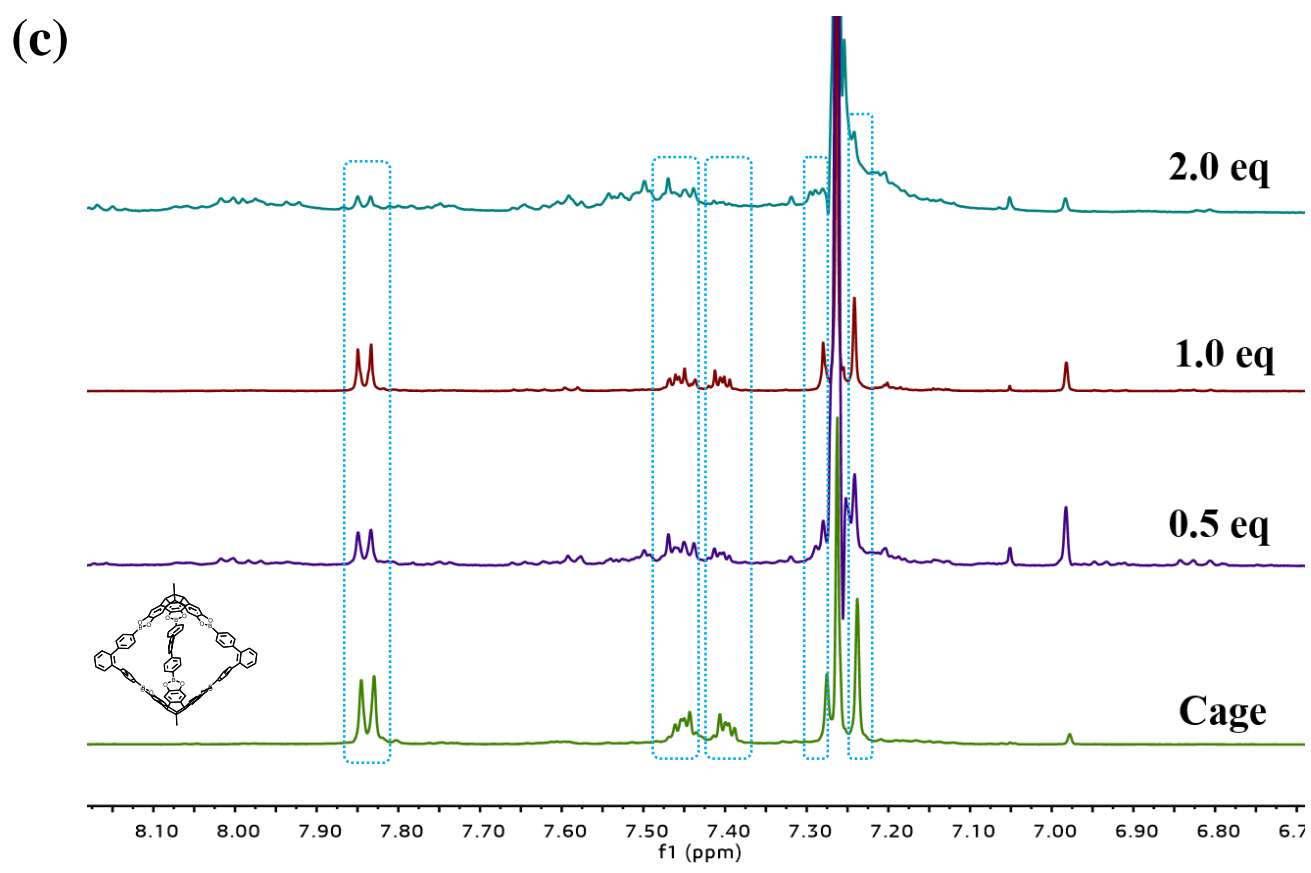

Figure S17. ${ }^{1} \mathrm{H}$ NMR spectra of (a) Cage-NUST-1 from COF-NUST-1; (b) Cage-NUST-1 from COF-NUST-2 and (c) the transformation from COF-NUST-1 to Cage-NUST-1 with different amount of TP monomer

\subsubsection{The transformation from Cage-NUST-1 to COF-NUST-1}

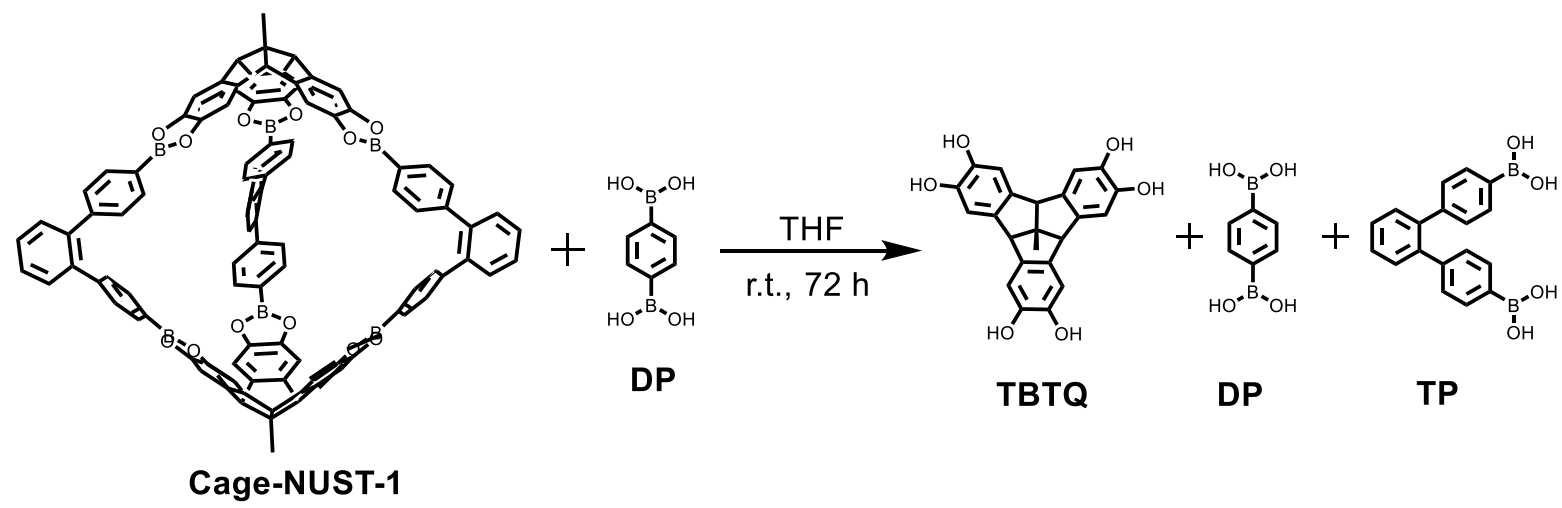

Cage-NUST-1 (40 mg, 0.026mmol), para-phenylene diboronic acid (13.1 mg, $0.079 \mathrm{mmol}$ ), anhydrous THF (4 mL) were added into a glass ampoule. A clear solution was obtained by ultrasound in 5 minutes. The glass ampoule was flash frozen at $77 \mathrm{~K}$ using the liquid nitrogen bath and degassed by freeze-pump-thaw three times, and then was sealed. The glass ampoule was placed at room temperature for three days, no precipitate formed. But when the glass ampoule was heated to $80{ }^{\circ} \mathrm{C}$ or $120^{\circ} \mathrm{C}$ for three days, a small amount of solid was collected as amorphous polymer, which was verified by ${ }^{1} \mathrm{H}$ NMR spectra and PXRD patterns respectively. 


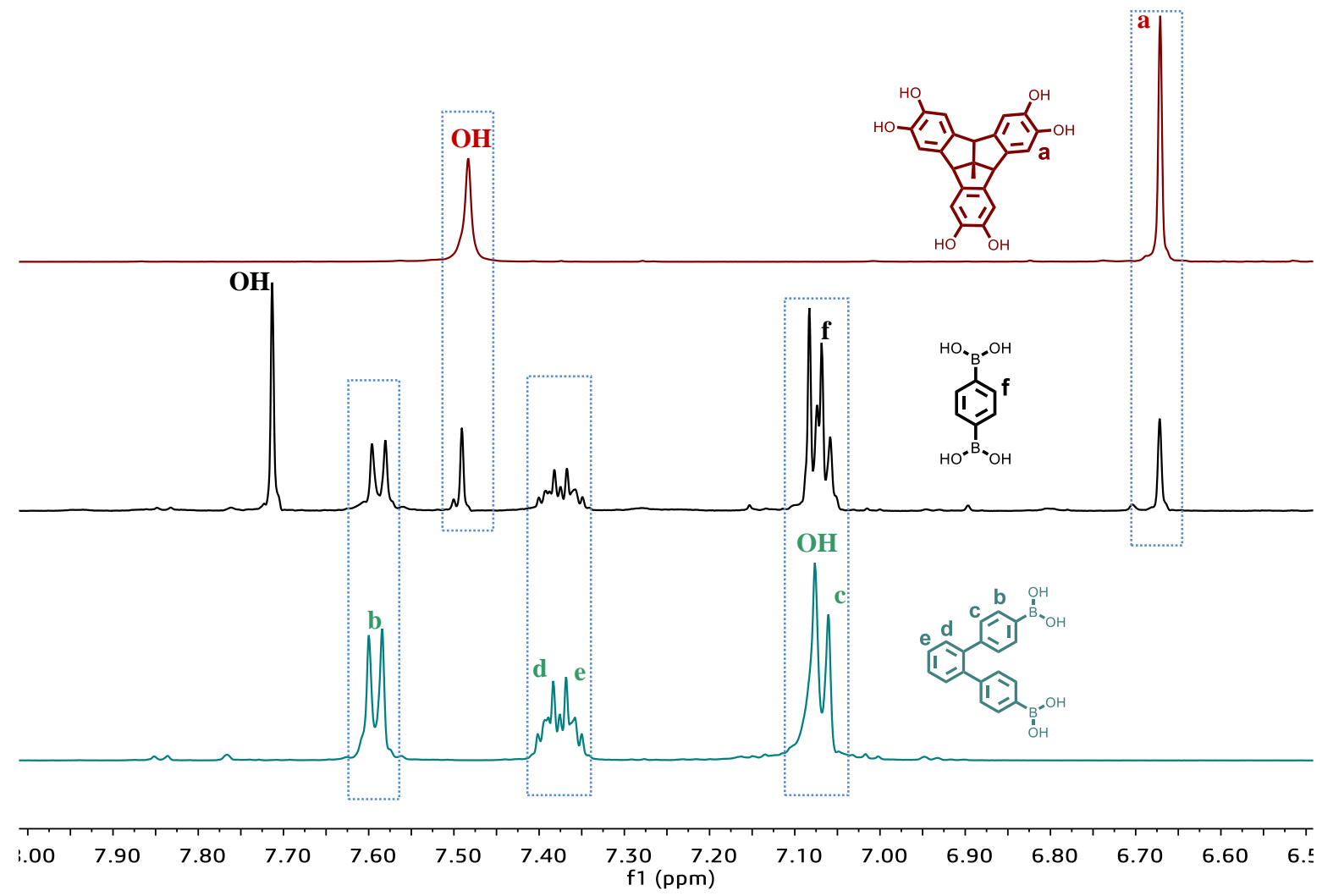

Figure S18. ${ }^{1} \mathrm{H}$ NMR spectra of the compounds from Cage-NUST-1 to COF-NUST-1 (black)

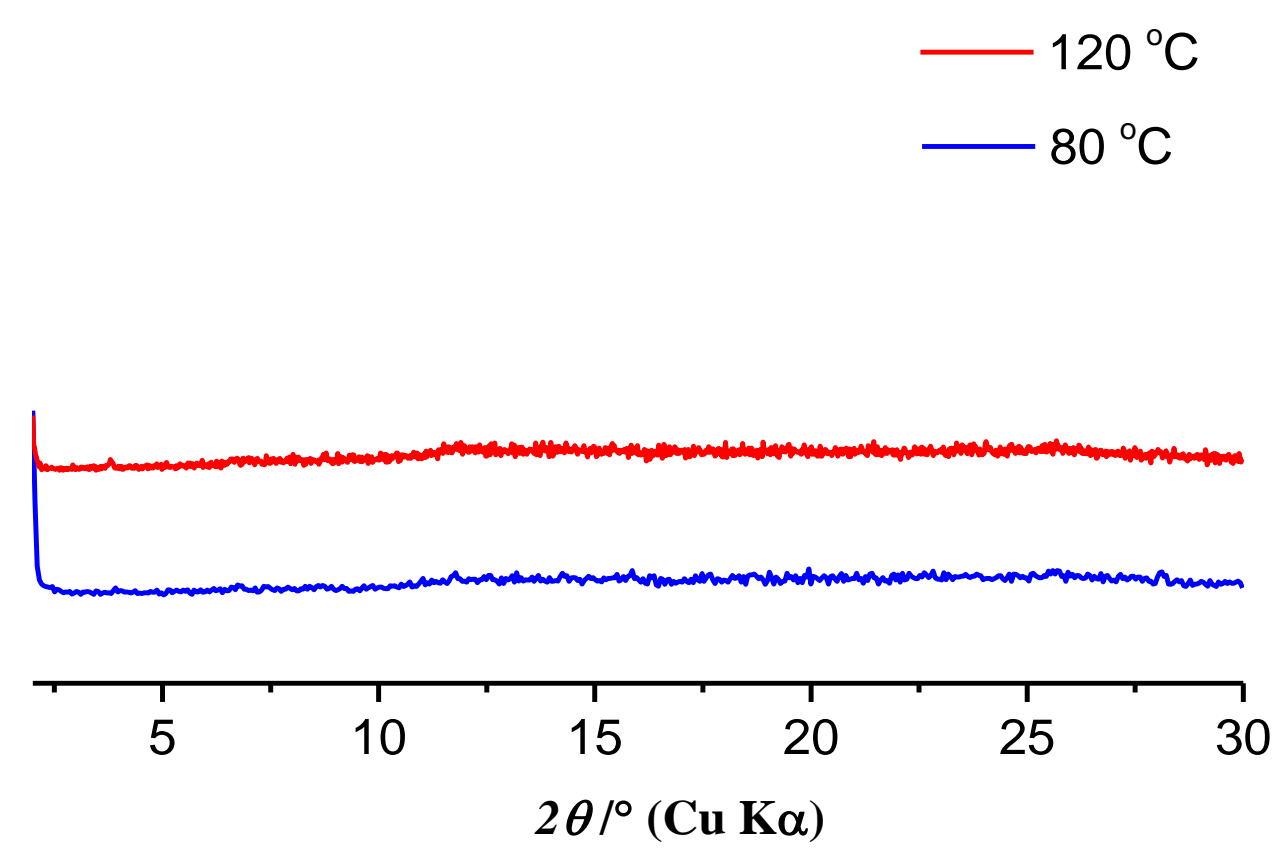

Figure S19. PXRD patterns of compound from Cage-NUST-1 to COF-NUST-1 at different temperature 


\subsubsection{Self-Sorting phenomenon in boron-oxygen Cage and COF}

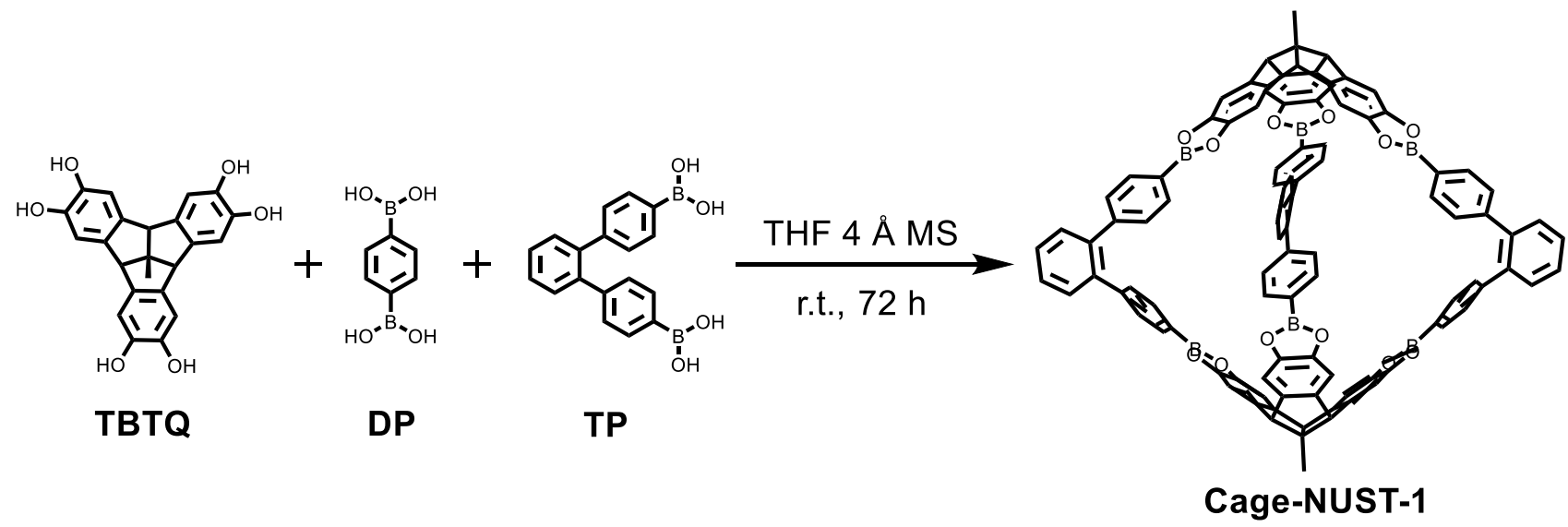

TBTQ (19.5 mg, $0.05 \mathrm{mmol})$, ortho-phenylene diboronic acid (23.9 $\mathrm{mg}, 0.075 \mathrm{mmol})$ and para-phenylene diboronic acid $(12.4 \mathrm{mg}, 0.075 \mathrm{mmol})$ were dissolved into a $20 \mathrm{~mL}$ glass vial with the addition of $4 \mathrm{~mL}$ anhydrous THF and molecular sieves $4 \AA$. The glass vial was placed at room temperature for three days, no precipitate formed. Cage-NUST-1 were afforded as characterized by ${ }^{1} \mathrm{H}$ NMR spectra of supernatant.

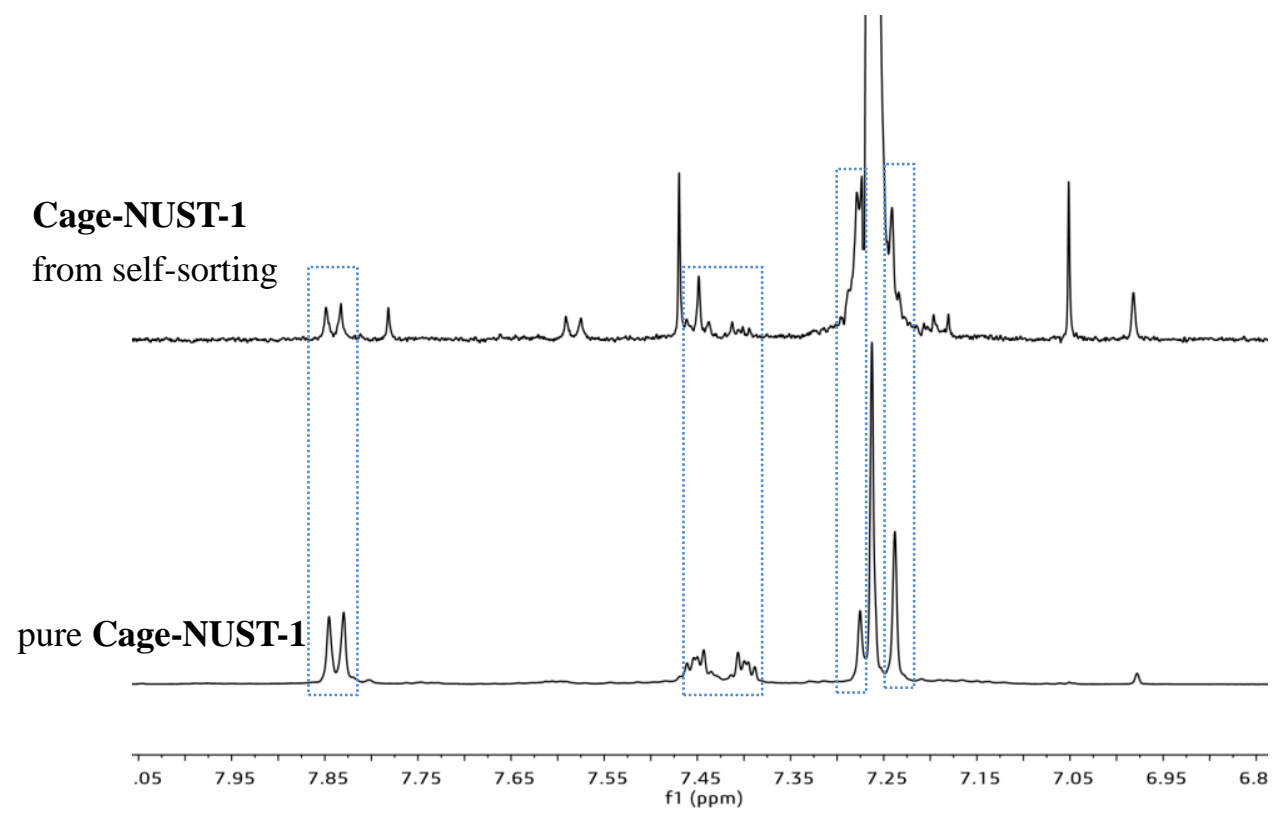

Figure S20. ${ }^{1} \mathrm{H}$ NMR spectra of Cage-NUST-1 from self-sorting 


\subsubsection{The transformation from Cage 1 to COF-LZU-1}

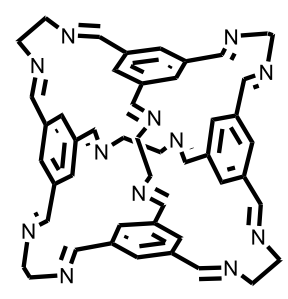

Cage 1

$+$<smiles>Nc1ccc(N)cc1</smiles>

PD

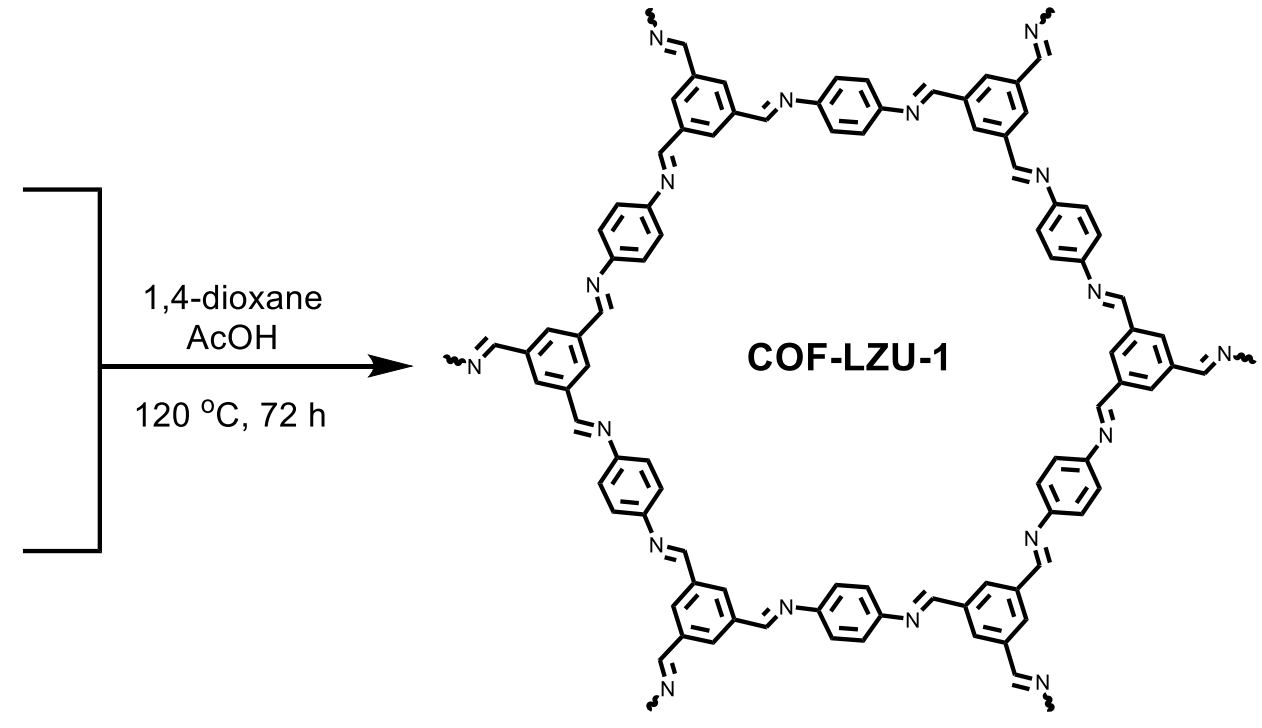

Cage 1 (30.0 mg, $0.038 \mathrm{mmol})$ and 1,4-diaminobenzene $(24.8 \mathrm{mg}, 0.23 \mathrm{mmol})$ were weighed into a glass ampoule with 1,4-dioxane ( $3 \mathrm{~mL})$. The solution was ultrasound for 5 minutes to obtain yellow turbid solution. $3 \mathrm{M}$ acetic acid $(0.6 \mathrm{~mL})$ was pure into the glass ampoule as catalyst. The glass ampoule was flash frozen at $77 \mathrm{~K}$ using the liquid nitrogen bath and degassed by freeze-pump-thaw three times, and then was sealed. The glass ampoule was placed at $120{ }^{\circ} \mathrm{C}$ for 3 days. The yellow solid was isolated by filtration and washed with 1,4-dioxane $(3 \times 10 \mathrm{~mL})$ and then filtered. The sample was then transferred to vacuum chamber and evacuated to $20 \mathrm{mTorr}$ at $50{ }^{\circ} \mathrm{C}$ for $24 \mathrm{~h}$, yielding yellow powder COF-LZU-1 (Yield: $26.2 \mathrm{mg}, 62 \%$ ). The experiment based on PD monomers' concentration has been accomplished, and the result shows that varying the concentration (0.5 2 equivalent of PD) cannot achieve the transformation efficiently (Figure S22b). 


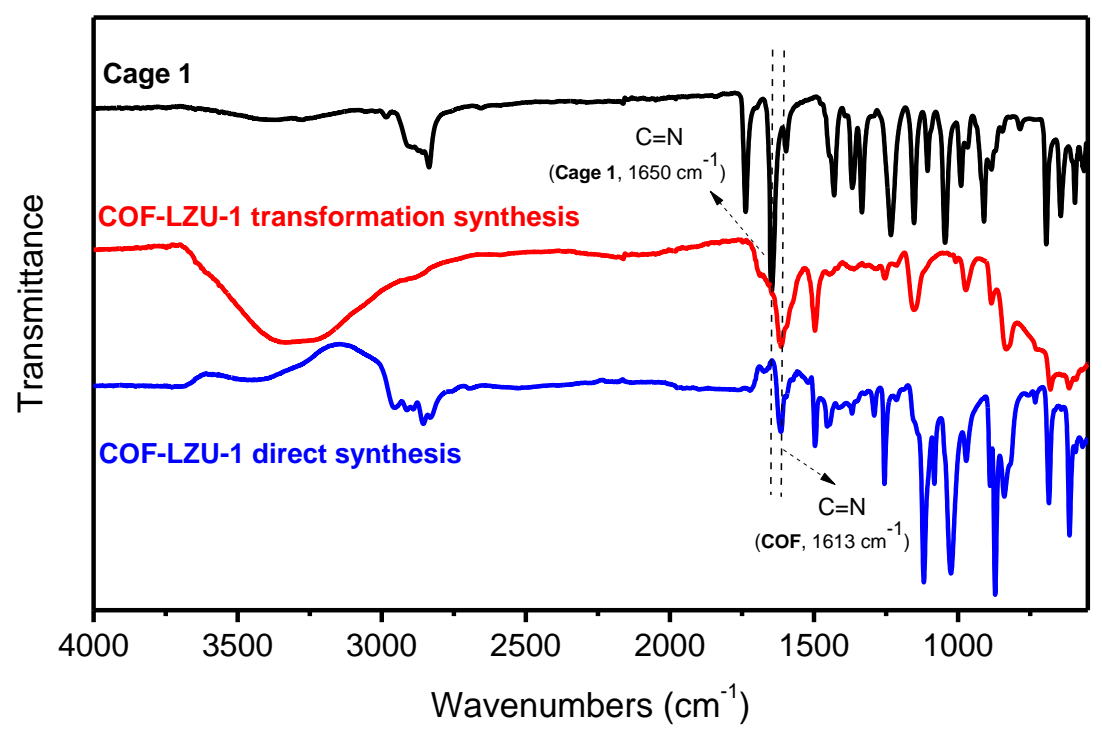

Figure S21. FT-IR spectra of Cage 1, COF-LZU-1 of transformation and direct synthesis

(a)
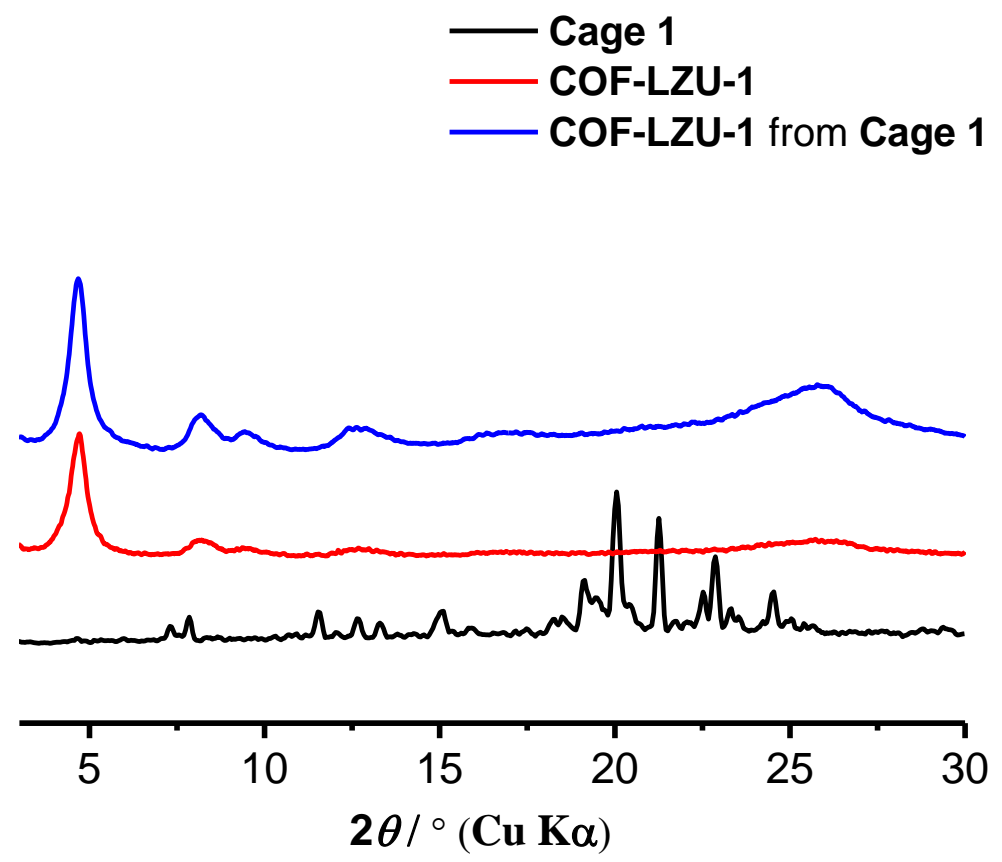
(b)
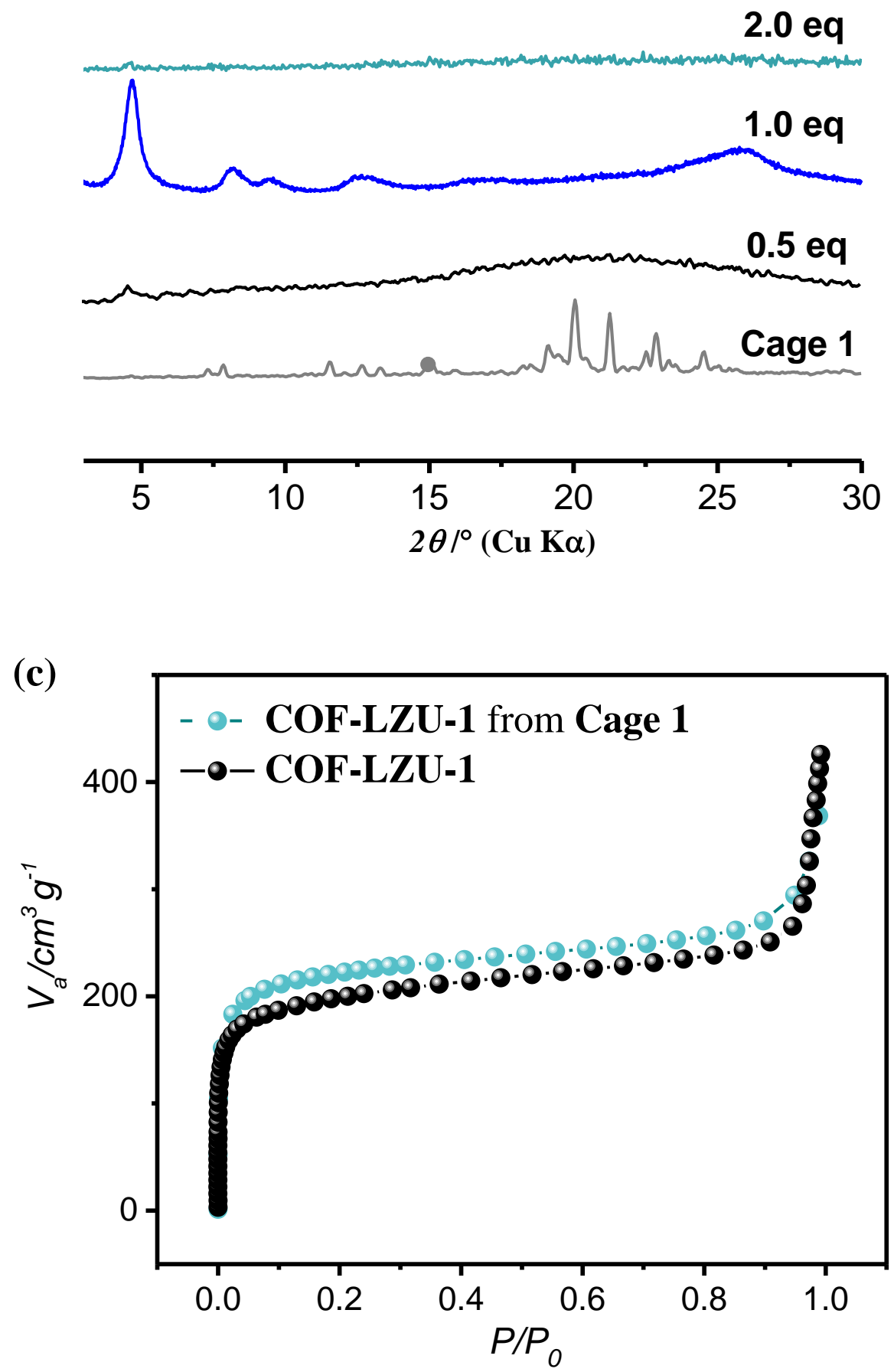

Figure S22. PXRD patterns of (a) COF-LZU-1 from Cage 1; (b) the transformation from Cage 1 to COF-LZU-1 with different amount of PD monomer and (c) Nitrogen adsorption curves of COF-LZU-1 


\subsubsection{The transformation from Cage 1 to COF-TFPA}

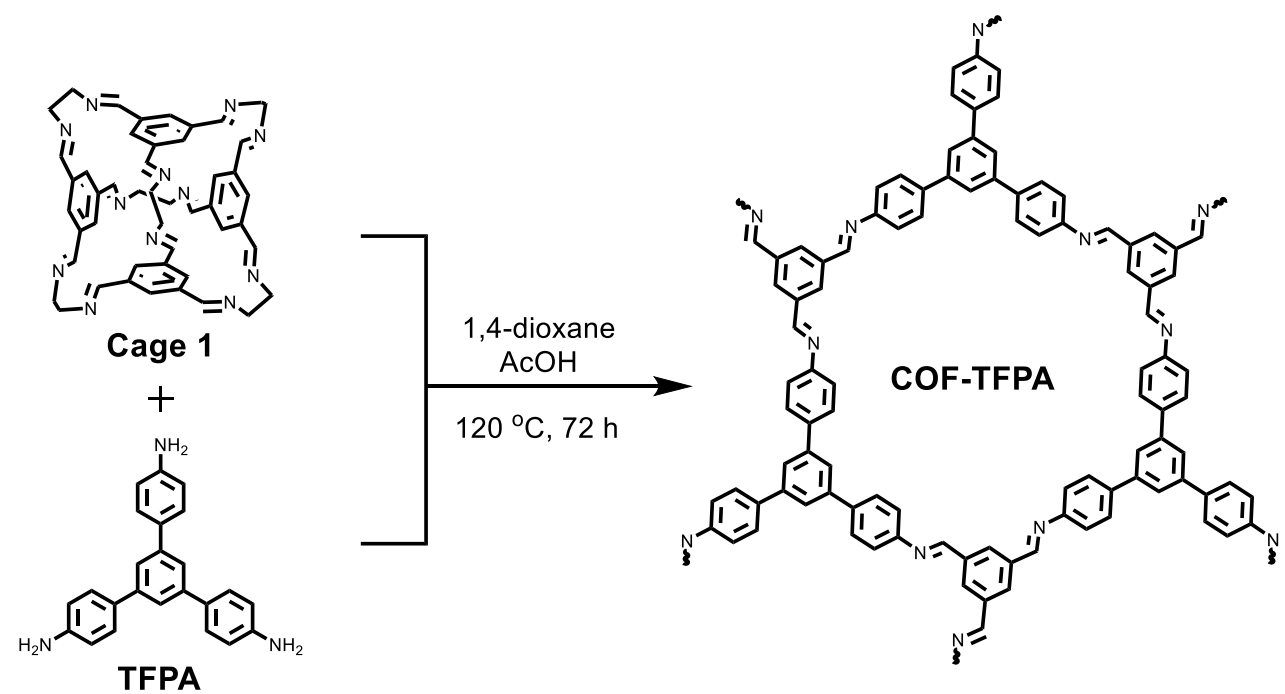

Cage $1(30.0 \mathrm{mg}, 0.038 \mathrm{mmol})$ and TFPA $(53.4 \mathrm{mg}, 0.15 \mathrm{mmol})$ were weighed into a glass ampoule with 1,4-dioxane ( $3 \mathrm{~mL}$ ). The solution was ultrasound for 5 minutes to obtain yellow turbid solution. $3 \mathrm{M}$ acetic acid $(0.6 \mathrm{~mL})$ was pure into the glass ampoule as catalyst. The glass ampoule was flash frozen at $77 \mathrm{~K}$ using the liquid nitrogen bath and degassed by freeze-pump-thaw three times, and then was sealed. The glass ampoule was placed at $120{ }^{\circ} \mathrm{C}$ for 3 days. The yellow solid was isolated by centrifugation and washed with 1,4-dioxane $(3 \times 10 \mathrm{~mL})$ and then filtered. The sample was then transferred to vacuum chamber and evacuated to $20 \mathrm{mTorr}$ at $50{ }^{\circ} \mathrm{C}$ for $24 \mathrm{~h}$, yielding yellow powder COF-TFPA (Yield: $52 \mathrm{mg}, 74 \%$ ).
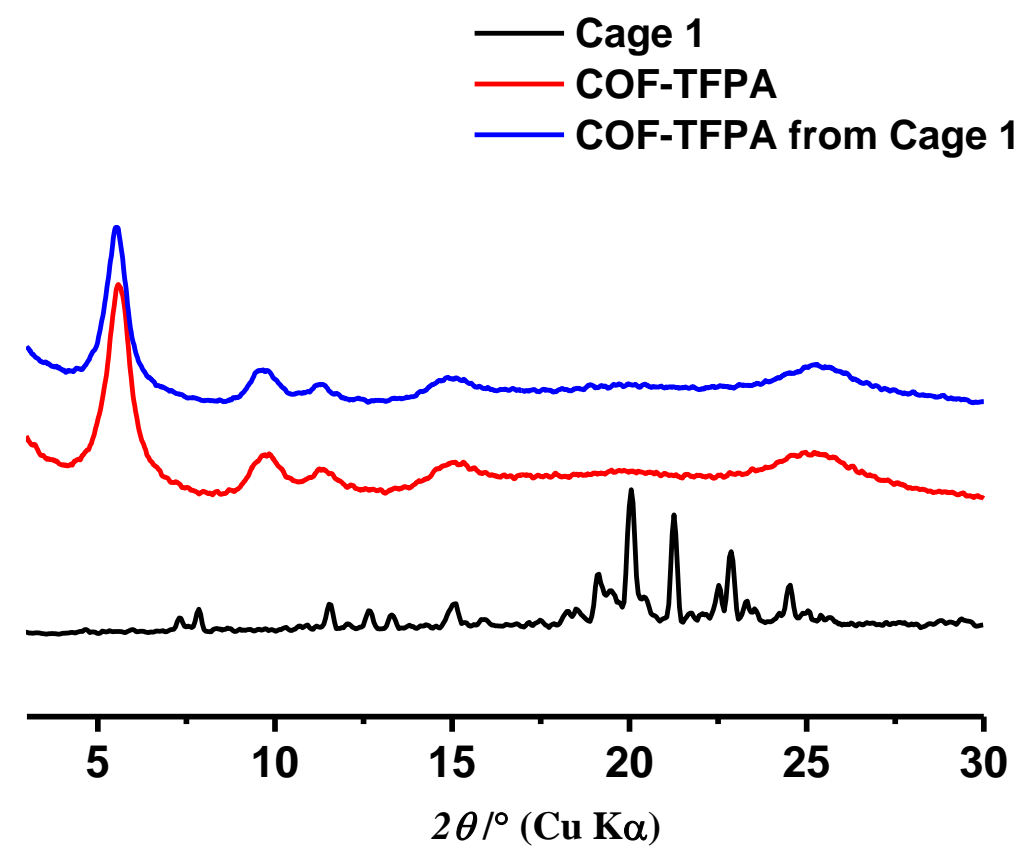

Figure S23. PXRD patterns of COF-TFPA from Cage 1 


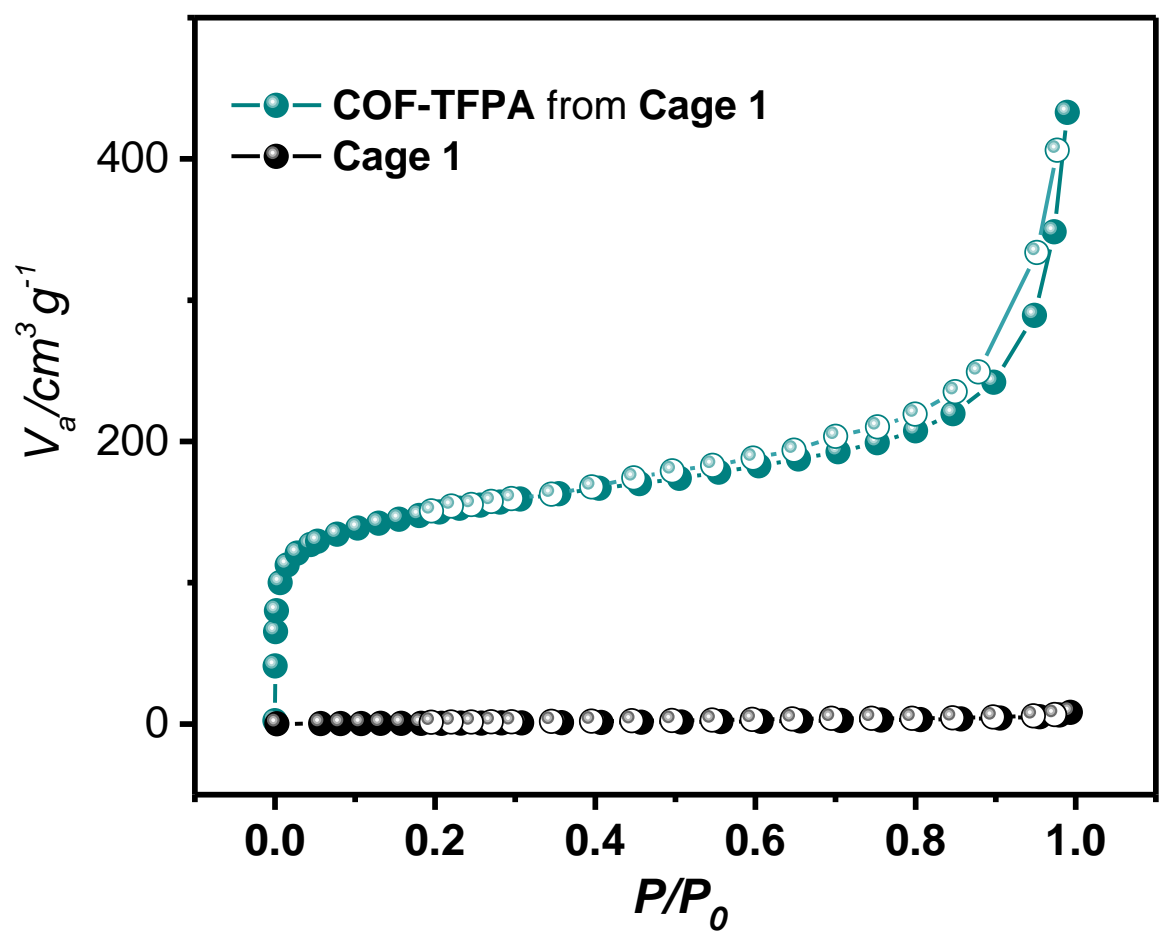

Figure S24. $\mathrm{N}_{2}$ adsorption-desorption isotherms of COF-TFPA from Cage 1

\subsubsection{The transformation from COF-LZU-1/TFPA to Cage 1}

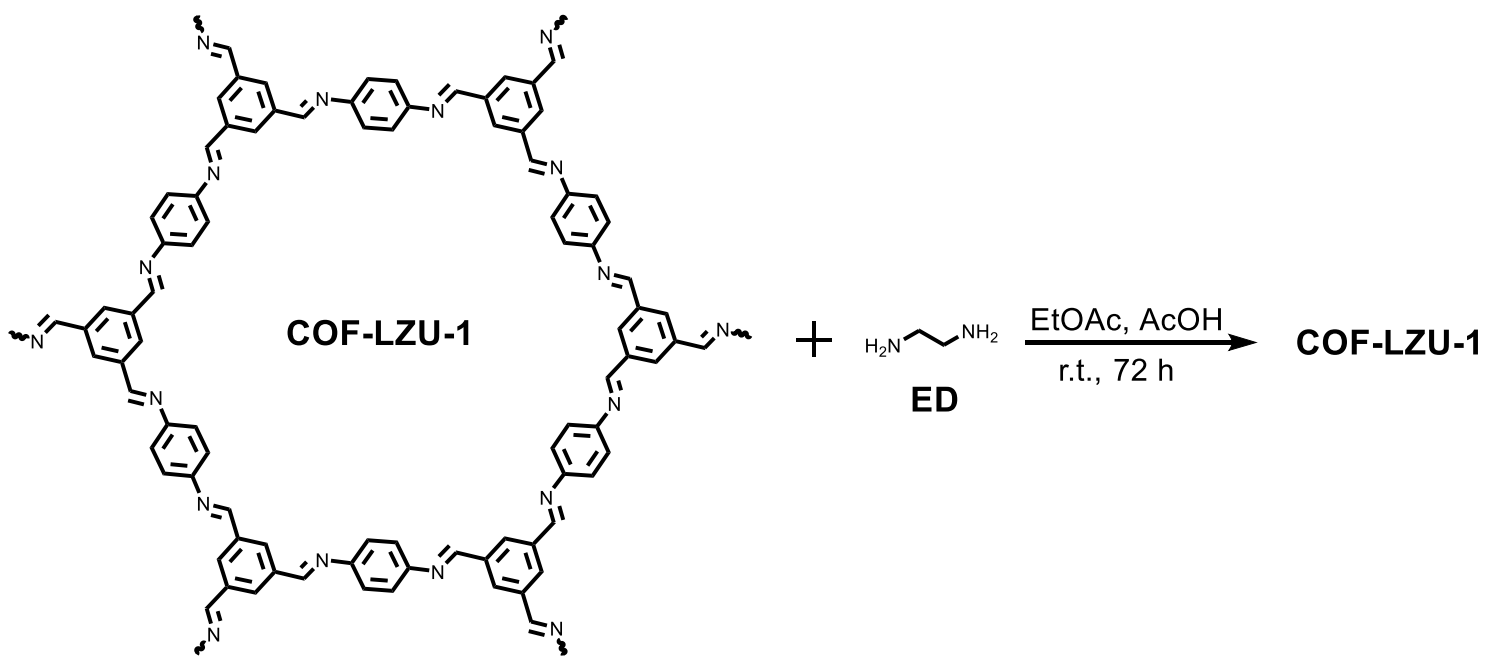

COF-LZU-1/TFPA (60 mg/240 mg) was weighed into a $50 \mathrm{ml}$ glass vial, EtOAc (35 mL) was added into the glass vial. Even with ultrasound, the COF is still insoluble. Then a solution of ethylene diamine $(31 \mu \mathrm{L}, 0.47 \mathrm{mmol})$ in EtOAc $(5 \mathrm{~mL})$ was added. After ultrasound for 5 minutes, 3 $\mathrm{M} \mathrm{AcOH}$ acetic acid $(0.6 \mathrm{~mL})$ was added. The glass vial was placed on a table at room temperature and left undisturbed for $72 \mathrm{~h}$. COFs remained as undissolved solid. The undissolved solid was collected through filtration, dried and tested by PXRD pattern. 
(a)

undissolved solid COF-LZU-1

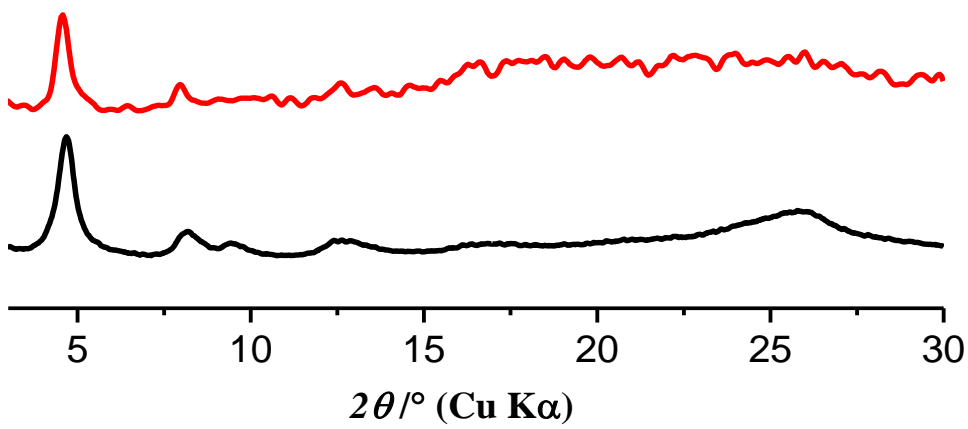

(b)

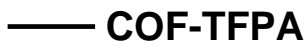

undissolved solid

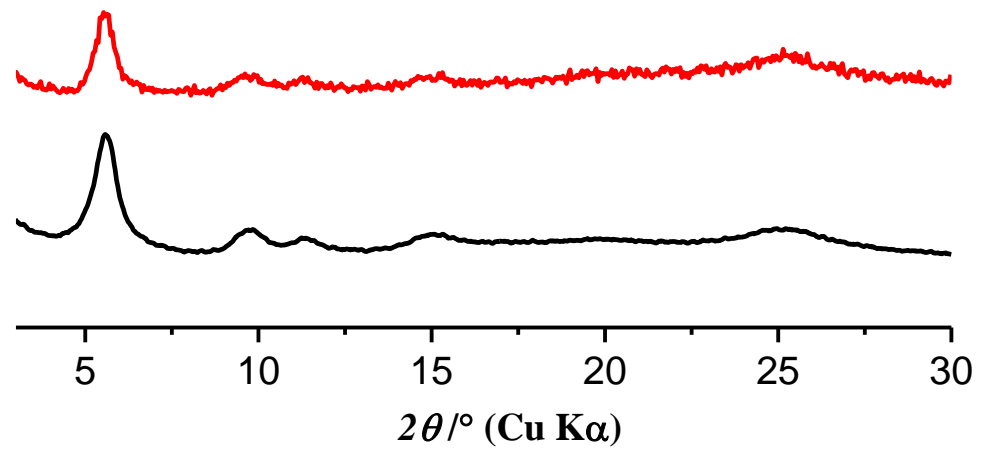

Figure S25. PXRD patterns of the transformation from (a) COF-LZU-1 to Cage 1; (b) COF-TFPA to Cage-1 


\subsubsection{Self-Sorting phenomenon in imine Cage and COF under cage's synthesis condition}

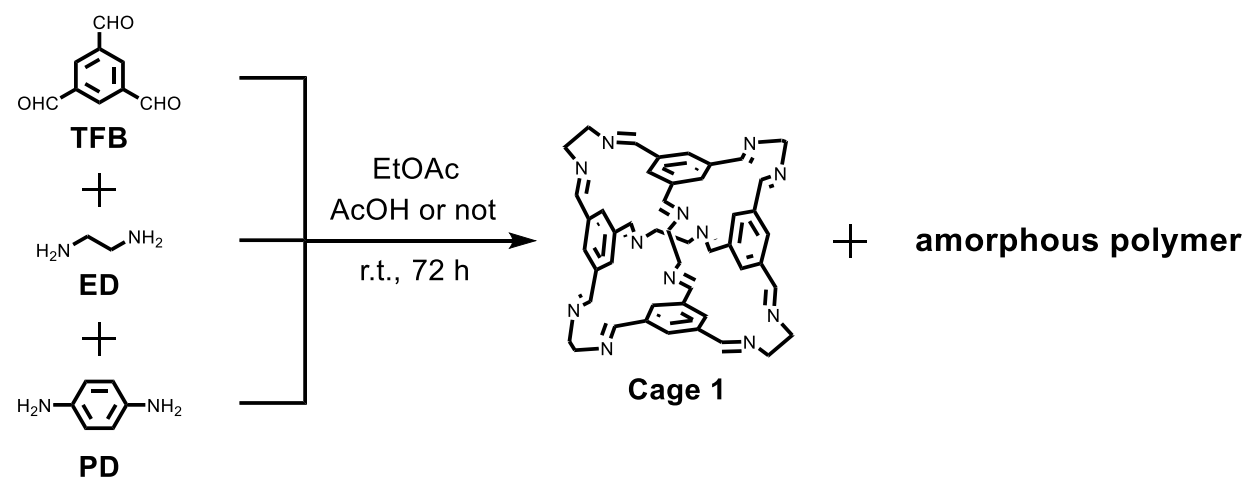

1,3,5-triformylbenzene (50 mg, $0.31 \mathrm{mmol})$, 1,4-diaminobenzene (49.7 $\mathrm{mg}, 0.46 \mathrm{mmol})$ were dissolved into a glass vial with Ethyl acetate $(35 \mathrm{~mL})$ at room temperature. Then a solution of ethylene diamine $(31 \mu \mathrm{L}, 0.47 \mathrm{mmol})$ in EtOAc $(5 \mathrm{~mL})$ was added. Two parallel reactions were performed, with or without acetic acid $(3 \mathrm{M}, 0.6 \mathrm{~mL})$ as the catalyst. The resulting mixture was left covered for $72 \mathrm{~h}$ without stirring. Finally, yellow sediment was observed in the solution and collected through filtration. The precipitate was partially dissolved in $\mathrm{CHCl}_{3}$ as Cage $\mathbf{1}$ which was confirmed by ${ }^{1} \mathrm{H}$ NMR in both reaction systems. The insoluble solid was proved to be amorphous polymer by PXRD patterns.

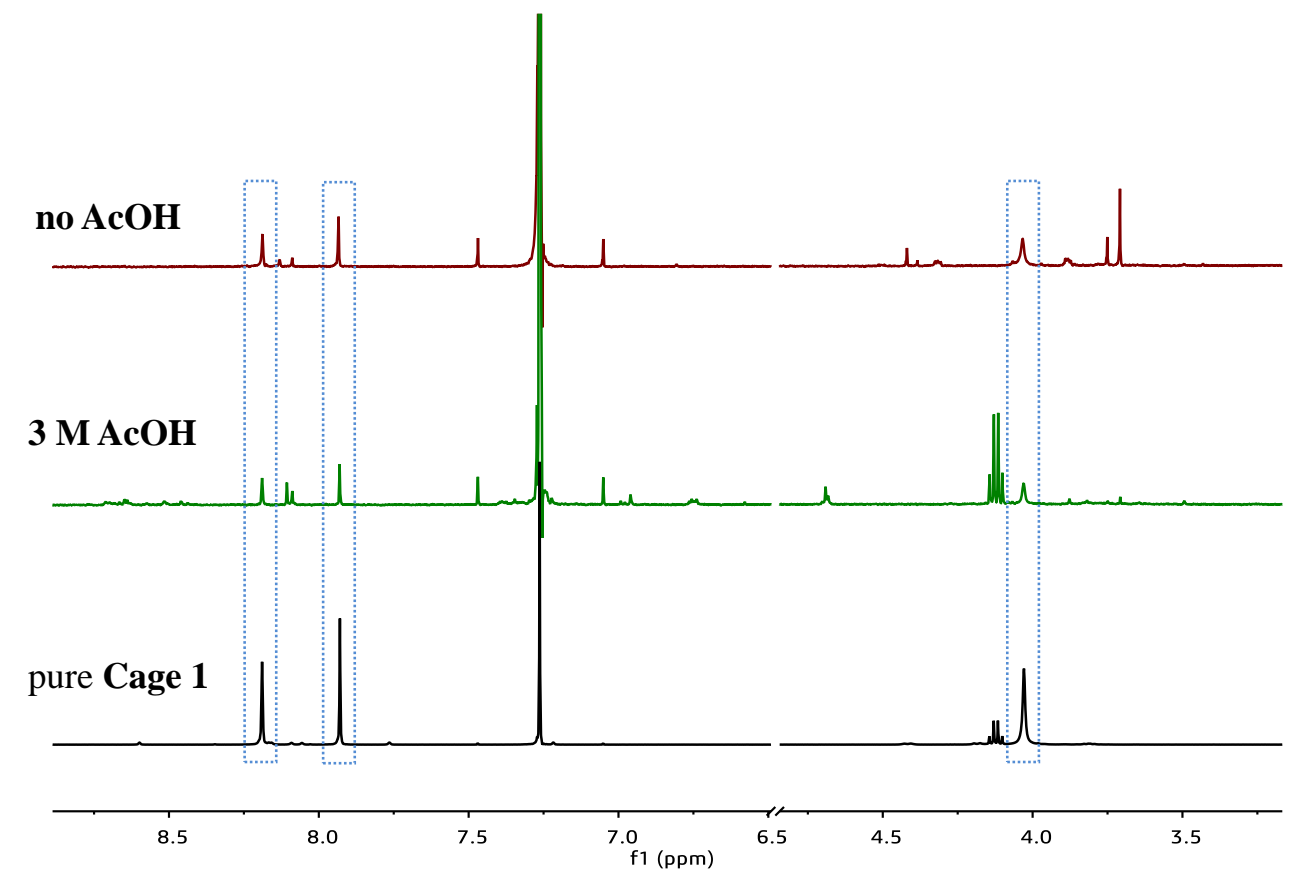

Figure S26. ${ }^{1} \mathrm{H}$ NMR spectra of Self-Sorting compounds Cage 1, with AcOH, without AcOH. 


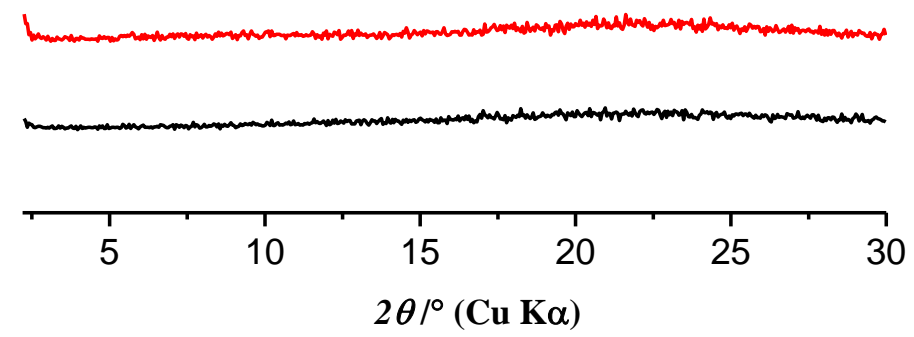

Figure S27. PXRD patterns of precipitate of Self-Sorting with/without the addition of AcOH.

\subsubsection{Self-Sorting phenomenon in imine Cage and COF under COF's synthesis condition}

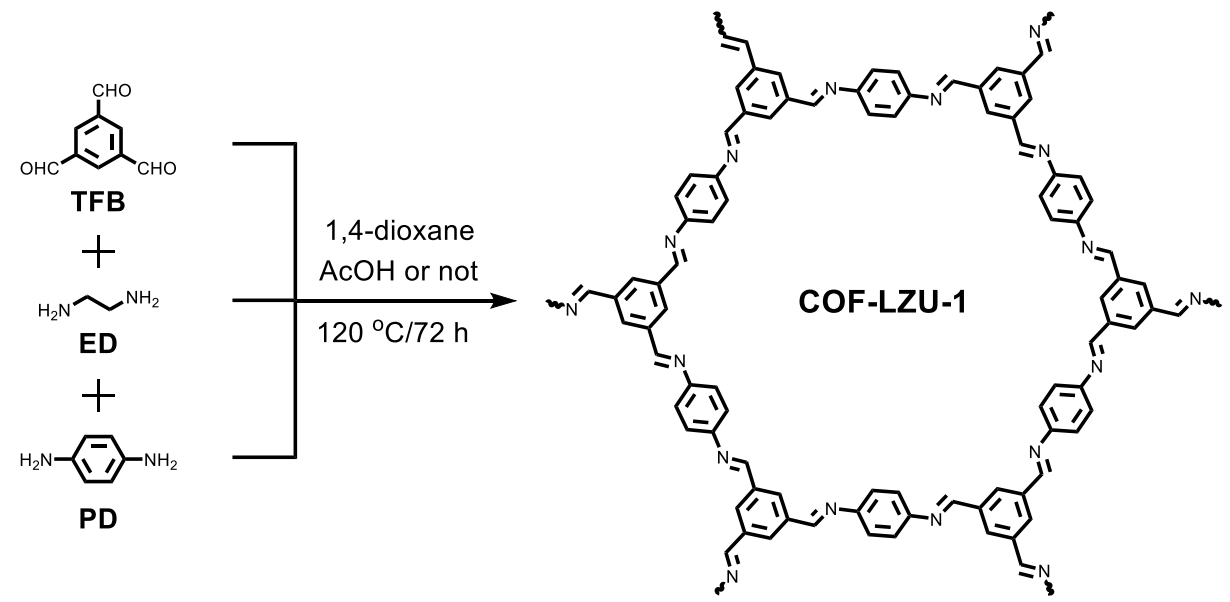

When the solvent was replaced by 1,4-dioxane and reactants were weighed into a glass ampoule containing 1,4-dioxane ( $3 \mathrm{~mL}$ ). The solution was ultrasound for 5 minutes to obtain yellow turbid solution. Two parallel reactions were also performed, with or without acetic acid $(3 \mathrm{M}, 0.6 \mathrm{~mL})$ as the catalyst. The glass ampoule was flash frozen at $77 \mathrm{~K}$ using the liquid nitrogen bath and degassed by freeze-pump-thaw three times, and then sealed. The glass ampoule was placed at $120{ }^{\circ} \mathrm{C}$ for 3 days. The yellow solid was isolated by filtration and washed with 1,4-dioxane $(3 \times 10 \mathrm{~mL})$ and then filtered. COF-LZU-1 was formed in the presence of acetic acid as catalyst which was confirmed by PXRD patterns. 


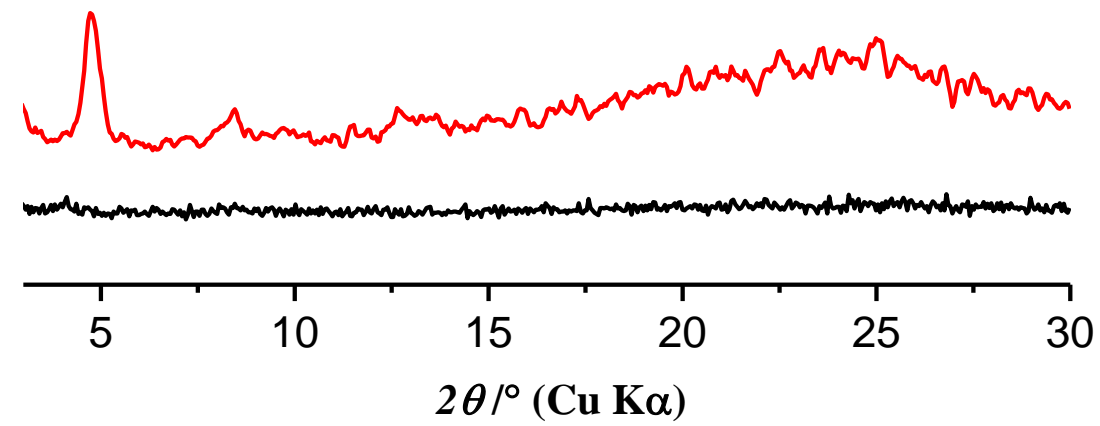

Figure S28. PXRD patterns of solid of Self-Sorting with/without the addition of $\mathrm{AcOH}$ at $120^{\circ} \mathrm{C}$

(a)

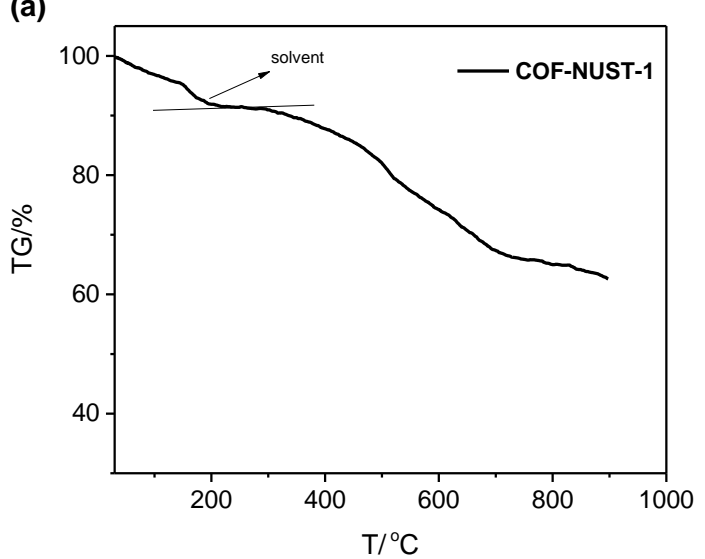

(c)

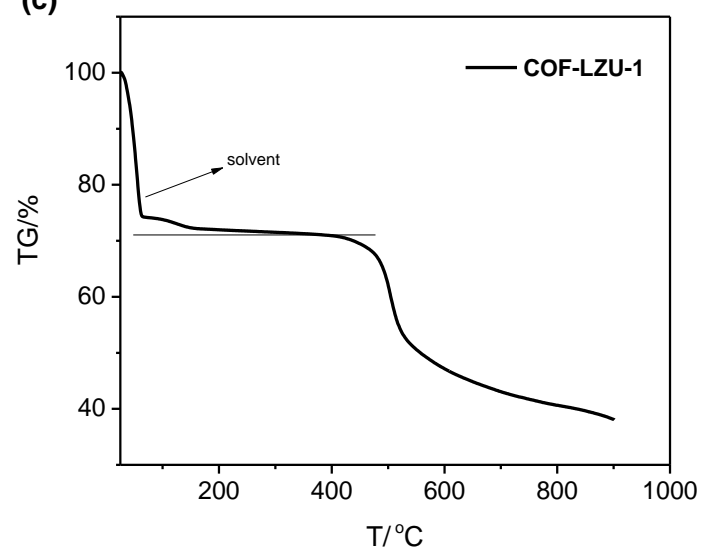

(b)

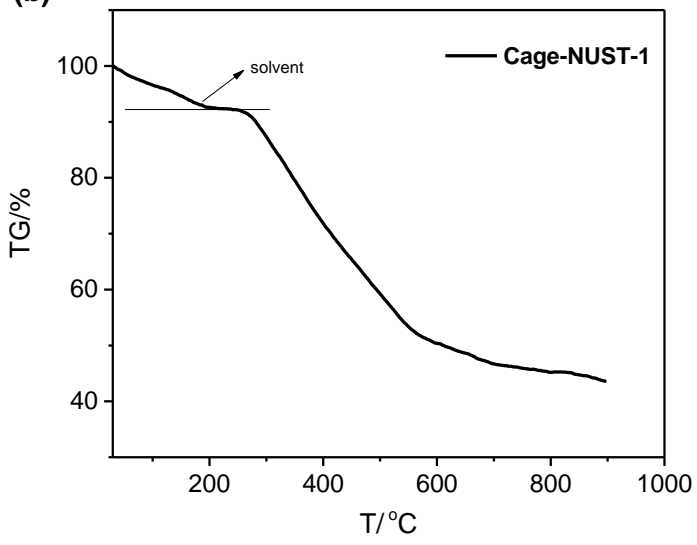

(d)

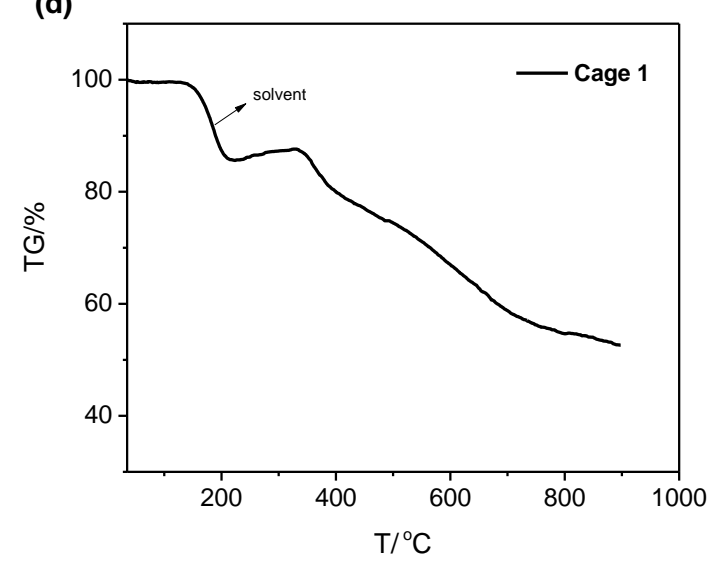

Figure S29. TGA curves of (a) COF-NUST-1; (b) Cage-NUST-1; (c) COF-LZU-1; (d) Cage 1 

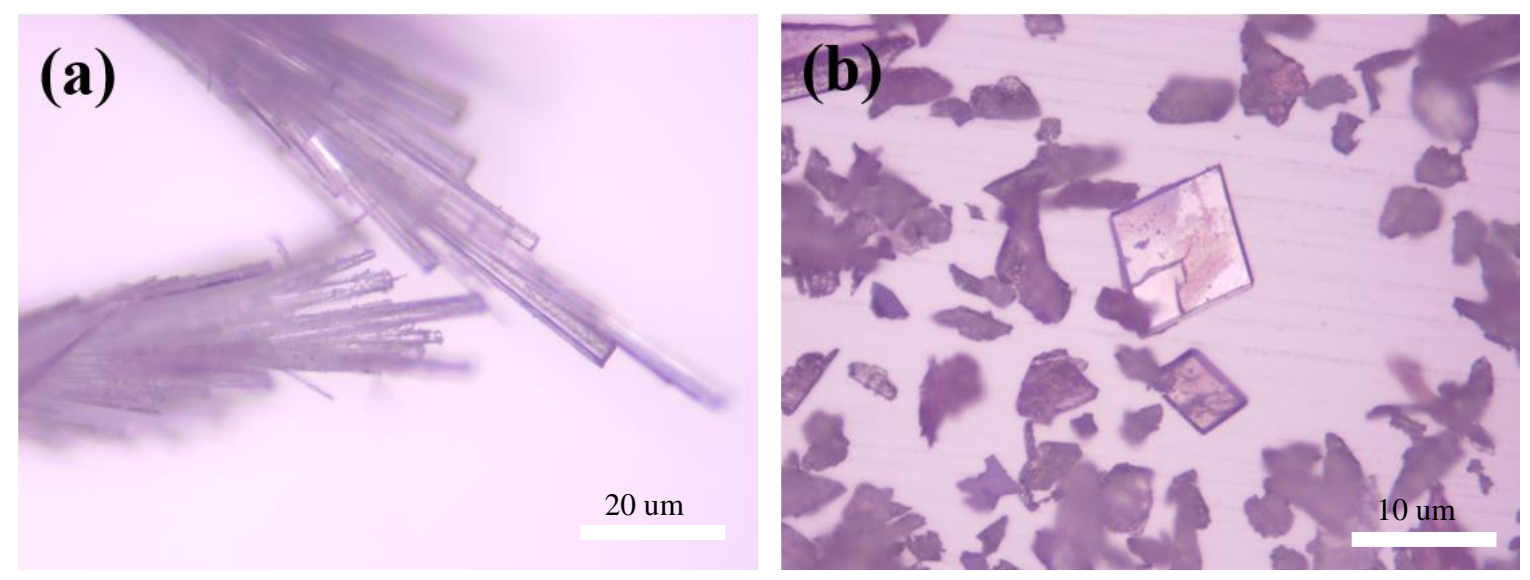

Figure S30. Optical microscope photograph of (a) Cage 1; (b) Cage-NUST-1
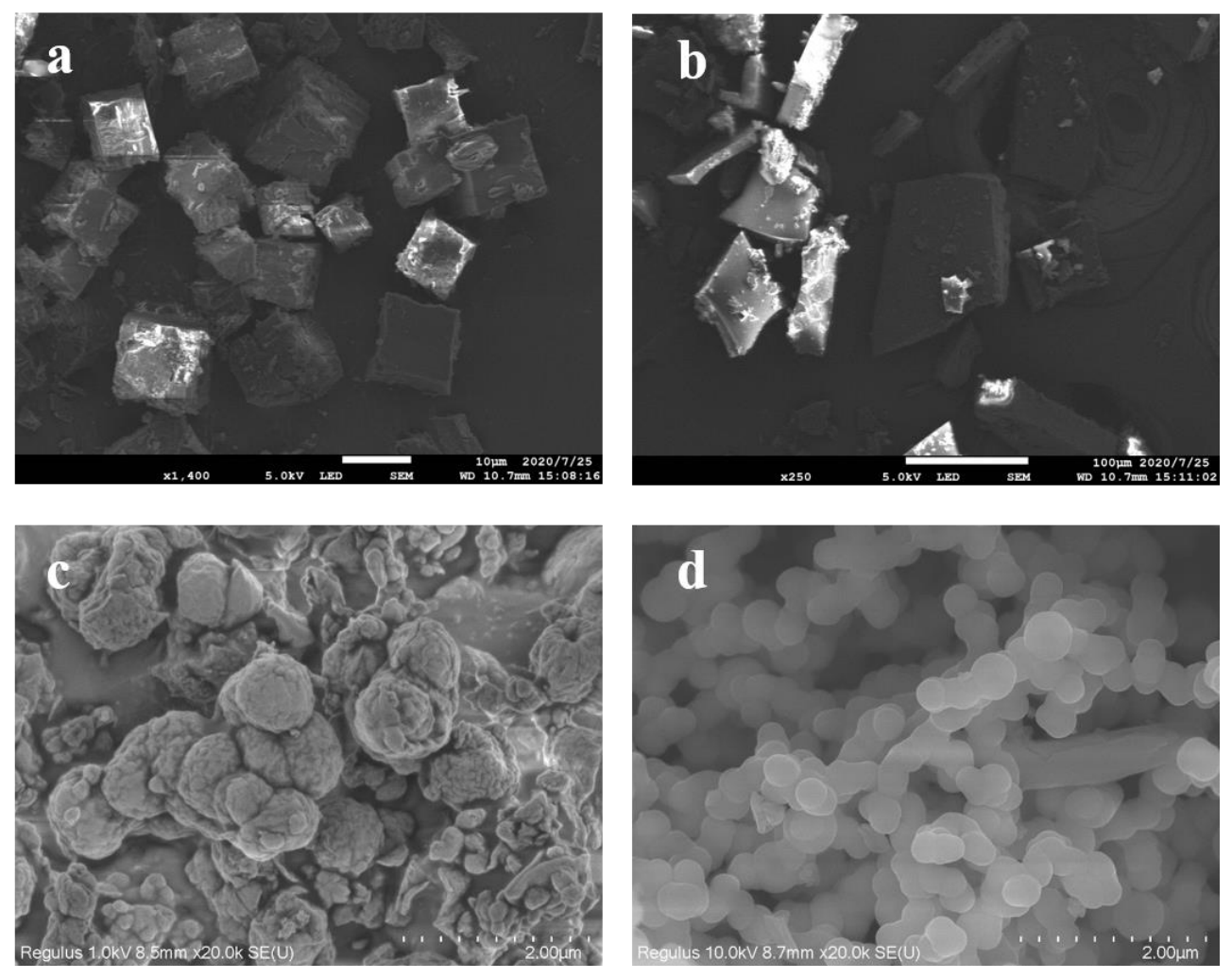

Figure S31. SEM images of (a) COF-NUST-1; (b) Cage-NUST-1; (c) COF-LZU-1; (d) Cage 1 

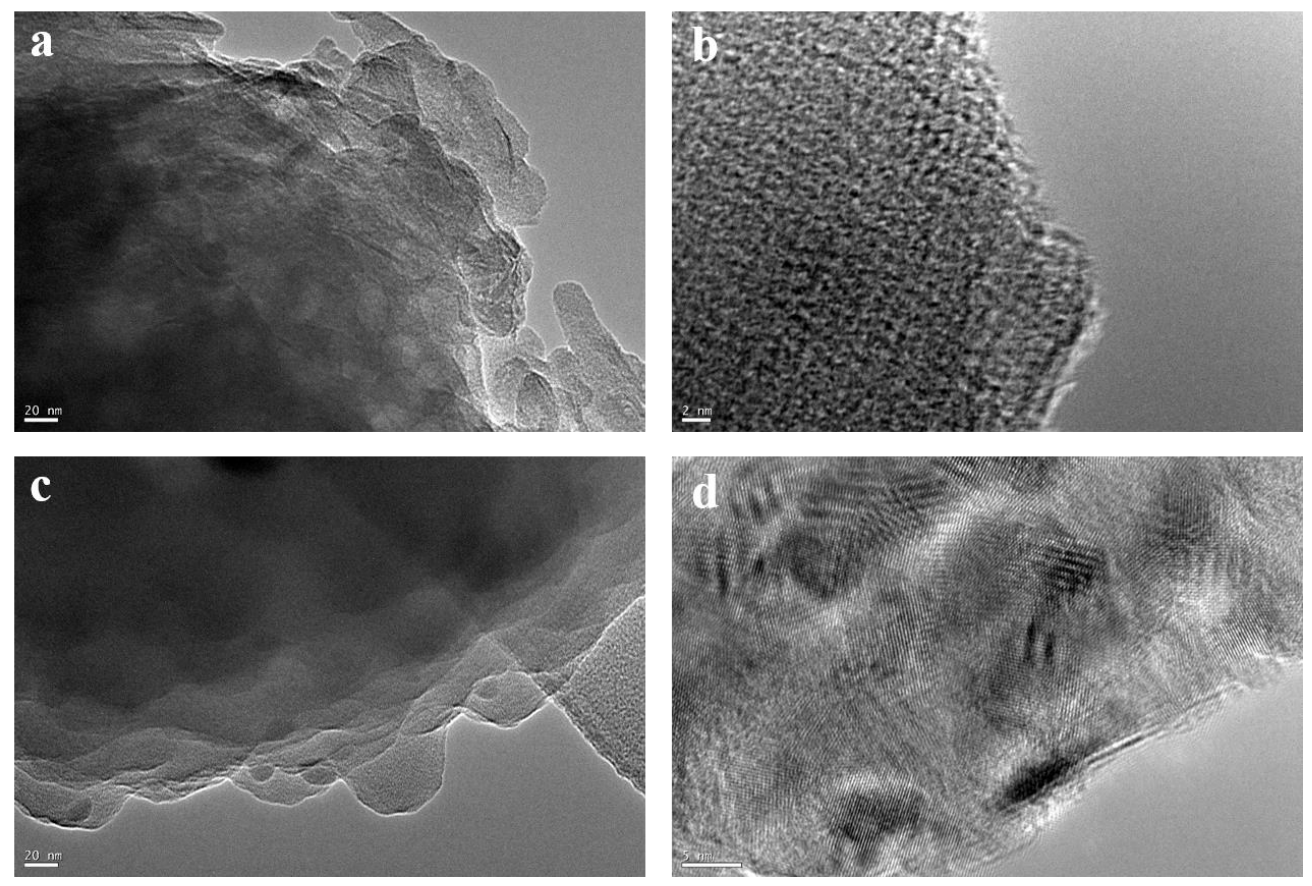

Figure S32. TEM images of (a, b) COF-NUST-1; (c, d) COF-LZU-1

\section{References}

(S1) He, L.; Ng, C.; Li, Y.; Liu, Z.; Kuck, D.; Chow, H.-F. Trefoil-Shaped Porous Nanographenes Bearing a Tribenzotriquinacene Core by Three-fold Scholl Macrocyclization. Angew. Chem. Int. Ed. 2018, 57, 13635-13639.

(S2) https://github.com/maddicoat/AuToGraFS

(S3) https://dftbplus.org/

(S4) http://www.ba.ic.cnr.it/softwareic/expo/

(S5) https://www.ill.eu/sites/fullprof/

(S6) http://www.zeoplusplus.org/

(S7) Zhang, G.; Tsujimoto, M.; Packwood, D.; Duong, N.; Nishiyama, Y.; Kadota, K.; Kitagawa, S.; Horike, S. Construction of a Hierarchical Architecture of Covalent Organic Frameworks via a Postsynthetic Approach. J. Am. Chem. Soc. 2018, 140, 2602-2609.

(S8) Romero-M, I.; Mavrandonakis, A.; Albacete, P.; Vega, A.; Briois, V.; Zamora, F.; Ana E. P-P. Unveiling the Local Structure of Palladium Loaded into Imine-Linked Layered Covalent Organic Frameworks for Cross-Coupling Catalysis. Angew. Chem. Int. Ed. 2020, 59, 13013-13020.

(S9) Tozawa, T.; Jones, J. T. A.; Swamy, S.-I.; Jiang, S.; Adams, D. -J.; Shakespeare, S.; Clowes, R.; Bradshaw, D.; Hasell, T.; Chong, S.-Y.; Tang, C.; Thompson, S.; Parker, J.; Trewin, A.; Bacsa, J.; Slawin, A. M. Z.; Steiner, A.; Cooper, A. -I. Porous organic cages. Nat. Mater. 2009, 8, 973-978. 